NIST GCR 18-015

\title{
Extending and Evaluating the Model-based Product Definition
}

\author{
Nathan W. Hartman \\ Jesse Zahner \\ Purdue University
}

This publication is available free of charge from:

https://doi.org/10.6028/NIST.GCR.18-015 
NIST GCR 18-015

\title{
Extending and Evaluating the Model-based Product Definition
}

\author{
Prepared for \\ Thomas D. Hedberg, Jr. \\ Allison Barnard Feeney \\ U.S. Department of Commerce \\ Engineering Laboratory \\ National Institute of Standards and Technology \\ Gaithersburg, MD 20899-8260 \\ By \\ Nathan W. Hartman \\ Jesse Zahner \\ PLM Center of Excellence \\ Purdue University
}

This publication is available free of charge from:

https://doi.org/10.6028/NIST.GCR.18-015

December 2017

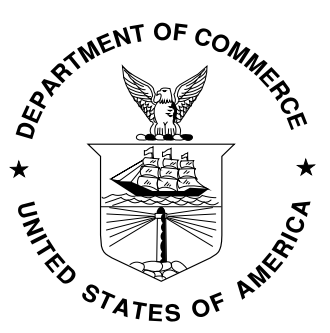

U.S. Department of Commerce Wilbur L. Ross, Jr., Secretary

National Institute of Standards and Technology Walter Copan, NIST Director and Undersecretary of Commerce for Standards and Technology 


\section{Disclaimer}

Any opinions, findings, conclusions, or recommendations expressed in this publication do not necessarily reflect the views of the National Institute of Standards and Technology (NIST). Additionally, neither NIST nor any of its employees make any warranty, expressed or implied, nor assume any legal liability or responsibility for the accuracy, completeness, or usefulness of any information, product, or process included in this publication.

The report was prepared under cooperative agreement 70NANB15H311 between the National Institute of Standards and Technology and Purdue University. The statements and conclusions contained in this report are those of the authors and do not imply recommendations or endorsements by the National Institute of Standards and Technology.

Certain commercial systems are identified in this report. Such identification does not imply recommendation or endorsement by the National Institute of Standards and Technology. Nor does it imply that the products identified are necessarily the best available for the purpose. 


\begin{abstract}
Industrial practice is in a state of transition, away from the use of drawings towards the use of annotated 3D CAD models as a means of communication. Working as a representation of an object or a system, a model-based product definition (MBD) is used to communicate information inside of a model-based enterprise (MBE). Such an enterprise will find itself in a transitional state as well, moving away from paper-based information sharing towards the use of modelbased, digital product data. This research investigation sought to identify the minimum information model (MIM) - the information elements necessary within an MBD to effectively employ a model as a replacement for a technical drawing in specific workflows in the product lifecycle. Over the course of this investigation, another phenomenon emerged - the common information model (CIM), which represents those information items that are necessary for the workflows targeted in this study. As expounded upon later in this report, the impacts of contextual domain knowledge on the implementation of the common information model is what formed the minimum information model discovered in this project.
\end{abstract}

\title{
Keywords
}

Model-Based Enterprise, Smart Manufacturing, Information Modelling 


\section{Contents}

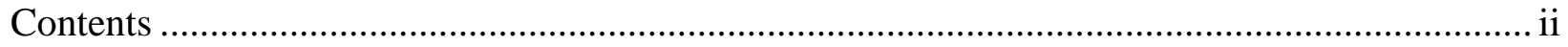

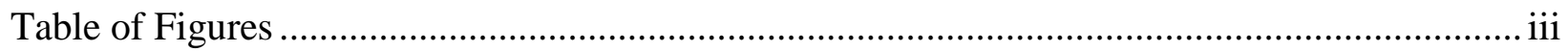

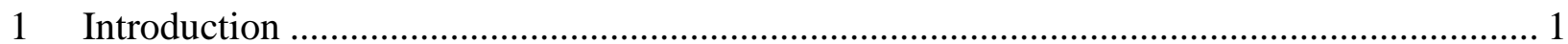

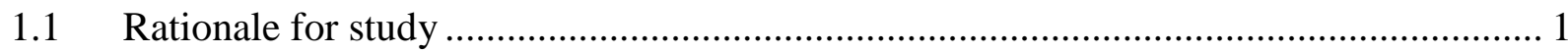

2 Background Literature Review.................................................................................... 3

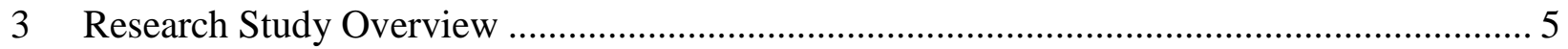

4 Stage One: Identifying Workflows and Demographics.......................................................... 6

5 Stage Two: Establishing the Importance of the MBD Information Items Using a Delphi

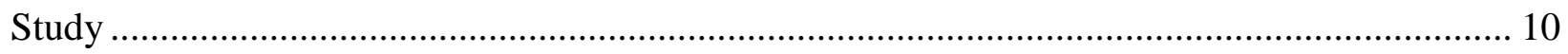

$5.1 \quad$ Delphi Survey Round One ....................................................................................... 11

5.2 Delphi Survey Round Two...................................................................................... 14

5.3 Delphi Survey Round Two Interview Data.............................................................. 18

5.4 Delphi Survey Round Three................................................................................... 20

5.5 Delphi survey Round Three Interview Data ............................................................. 24

6 Stage Three: Using IDEF0 Models to Contextualize the Minimum Information Model...... 26

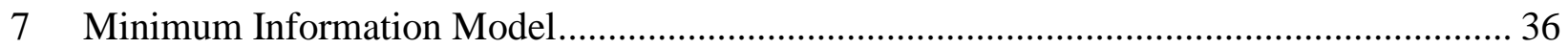

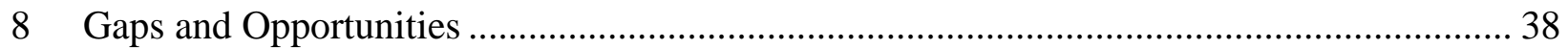

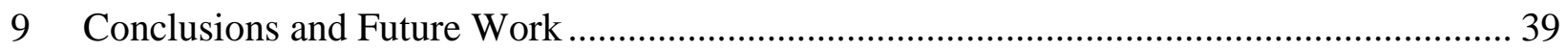

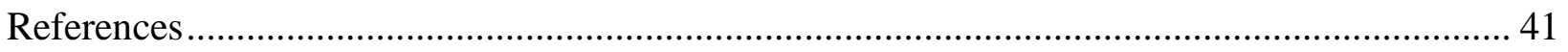

Appendix A: Survey Questions for Stage One ............................................................................. 43

Appendix B: Delphi Study Round 1 Questions ........................................................................ 50

Appendix C: Delphi Round Two ..................................................................................... 56

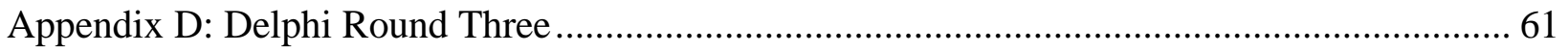

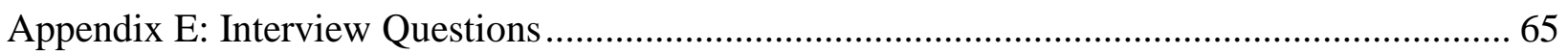




\section{Table of Figures}

Figure 1: Distribution of Industry Participation (N: 76) ......................................................... 7

Figure 2: How participants receive information (N: 68) ......................................................... 8

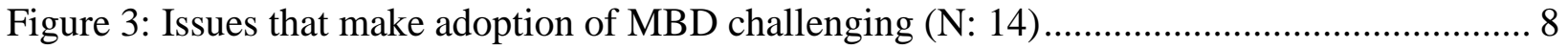

Figure 4: What information was created or used in the product information for the Concept to

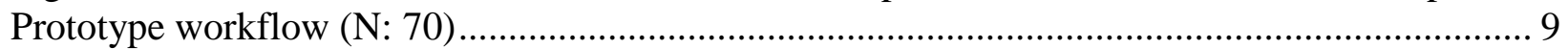

Figure 5: Could models be used in place of drawings in your workflow? (N: 50)........................ 9

Figure 6: Assuming you use a MBD across the lifecycle of your products, which elements are common from one life cycle stage to the next? (Select all that apply) N:46 .

Figure 7: To what extent does your job role involve the use of a drawing or model to complete your job effectively? N: 23 ............................................................................................... 12

Figure 8: Which industry sector best represents your company, or the division of the company

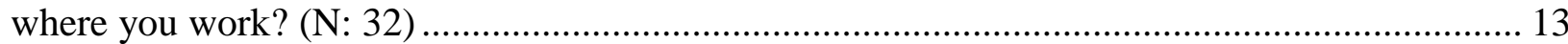

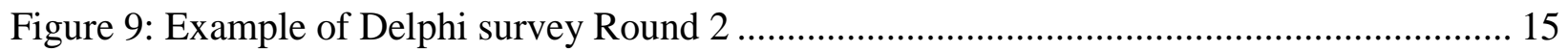

Figure 10: Q1 - Which industry sector best represents your company or the division of the

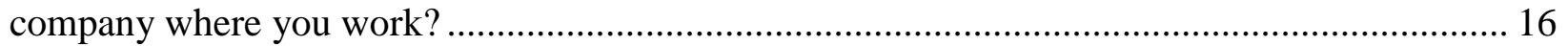

Figure 11: ANOVA data for Delphi survey Round 2 ......................................................... 18

Figure 12: Q2 - Which industry sector best represents your company or the division of the

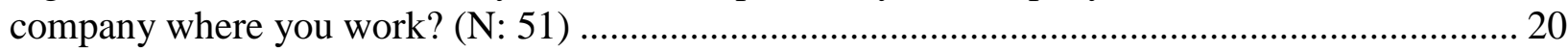

Figure 13: Questions 6-9 on Element Rating (N: 49-50).......................................................... 21

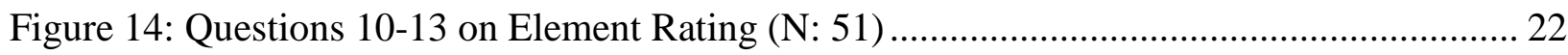

Figure 15: Questions 13-17 on Element Rating (N: 49-50)........................................................ 22

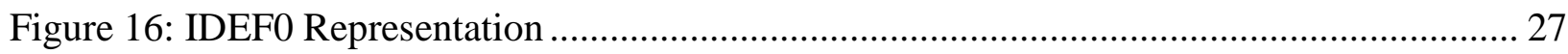

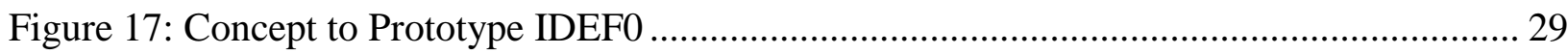

Figure 18: Concept to Prototype IDEF0 Revised ..................................................................... 30

Figure 19: Prototype to Detailed Product Definition........................................................................ 31

Figure 20: Prototype to Detailed Product Definition Revised........................................................... 31

Figure 21: Detailed Product Definition to Manufacture ............................................................... 33

Figure 22: Detailed Product Definition to Manufacture Revised .................................................. 33

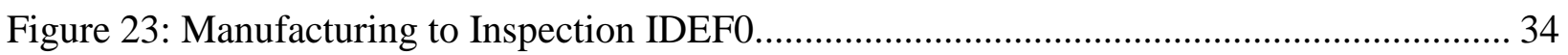

Figure 24: Manufacturing to Inspection IDEF0 Revised........................................................... 35

Figure 25: Minimum Information Model Diagram ................................................................ 37

Figure 26: Primary and Auxiliary Information ......................................................................... 38 


\section{Introduction}

Industrial practice is in a state of transition, away from the use of drawings towards the use of annotated 3D CAD models as a means of communication. Working as a representation of an object or a system, a model-based product definition (MBD) is used to communicate information inside of a model-based enterprise (MBE). Such an enterprise will find itself in a transitional state as well, moving away from paper-based information sharing towards the use of model-based, digital product data. Being able to leverage the communicative power and the depth off information provided by the MBD requires an understanding of the information needs of the various authors and consumers of product information across the enterprise. However, critical information stored in the digital product definition is often lost in translation or not explicitly defined in a way that users in the communications processes can consume it effectively.

Historically, technical drawings contained much more information than just the geometric and dimensional information for an object. They contained information about materials, steps for the assembly process, revision or version history, production process information, and many other bits of information that were implicitly defined based on the context in which the drawing was used. In current industrial practice, much of that same information is not captured in a digital model, even though a model is often considered a replacement for drawings. Just as the practice for creating technical drawings was loosely governed by a company's adherence (or not) to published standards, so is the case with the creation of modelbased definitions. Yet, manufacturing companies often express that they have a goal to use the communicative power and accuracy of an MBD across the enterprise, especially the supply chain, but there is a lack of understanding and agreement for what data is to be included in an MBD. Thus, the development and enforcement of standards is difficult, and the ability to document a return on investment for switching to a model-based definition approach is tenuous.

This research investigation sought to identify the minimum information model (MIM) - those information elements necessary within an MBD to effectively employ that model as a replacement for a technical drawing in each workflow. Over the course of this investigation, another phenomenon emerged - the common information model (CIM), which represents those information items that are necessary for the workflows targeted in this study. As expounded upon later in this report, the impacts of contextual domain knowledge on the implementation of the common information model is what formed the minimum information model discovered in this project.

\subsection{Rationale for study}

The creation of 3D CAD models typically focuses on the capture of accurate shape definition by using explicit dimensional and geometric constraints, parametric features, and relational database functionality. However, there is more to the design and manufacture of an object than those items. When technical drawings were used as a communications mechanism, material characteristics, process information, inspection data, and other specifications were often 
captured in a way that is impossible to do with modern CAD tools. The inability to capture behavioral and contextual information centers on the required use of explicit definition of constraints for shape definition in contemporary CAD software tools. Behavioral and contextual information in manufacturing environments, specifically design and production scenarios, is often understood by humans working in the environment without the need for specific detail or definition. Due to what was typically a lack of space on a drawing sheet, and the need to make text on a drawing precise and concise, human operators came to understand specific symbology and terminology in the context of a company's environment. The relative inability to accurately capture the same set of semantics from a drawing within the 3D model has led to much confusion in recent years within the industrial community around which information items from a drawing should be included in the creation of a model-based definition.

The move towards the model being the master definition is difficult because there are many things that stand in the way. One such thing is the information that is communicated across the enterprise. Product information standards, such as ASME Y14.41 [1], provide guidance for presenting information in a MBD, as well as how to facilitate its creation within an authoring tool. However, the standards do not tell the user what information to present at specific stages of the lifecycle or even inside of their specific information workflows [2].

Product-related communication does not rest solely with the engineering or design teams, and as such, elements of MBD need to be properly communicated or expressed in all stages of the lifecycle. However, the elements that should be expressed outside of the design stage are still open to debate. Information elements expressed outside of the design stage and its focus on geometry are especially important for companies that have chosen to transform into a MBE. $\mathrm{MBE}$ is an organization utilizing information in its operational functions provided by models, especially in the making of decisions regarding the product lifecycle. A model-based enterprise leverages the information provided in the 3D model to communicate, collaborate, create, and support the product. A model-based enterprise will use this information and embrace any changes needed to improve the representation of their digital product information and supporting architecture, and these changes or improvements should resonate in all future iterations of that product or architecture, and will compound in time to create a more effective product.

Authors and consumers of product information from across the enterprise do not need the same information. Those internal to the enterprise have differing needs for information based on their role and position than those outside the organization. To compensate for the information needed by different individuals, the product definition must change or be translated to provide different amounts and types of information depending on the needs of the information consumer. Upon translation or modification of the model for any given consumer, there is a high likelihood for information loss. Moreover, the more information added to the model during creation, the more difficult it is to validate that a translation or modification occurred correctly [3].

Prior to the existence of 3D models and the concept of a model-based definition, information loss based on data exchange did not exist in the same way. Drawings contained both implicit and explicit information, which when being passed from one individual to the next was not at risk of being lost or miscommunicated, since the form of the communication medium (i.e., 
paper) did not change [4]. Consumers of the drawing simply interpreted the drawing based on their own semantic context of the object in question. The data could also be interpreted incorrectly. Different users will have different takes on the data. An engineer and a manager will not look at the data the same way. A major problem could arise when taking implicit or explicit information into account, as managers will not know what information needs to be assumed, similarly engineers or designers may not always grasp all implied information. Again, if models are to effectively replace drawings as a communications medium, what goes into the model must be able to come out in a form that can be understood and used [5].

The accurate exchange of information becomes imperative when having to share the product definition with persons outside of the model-based enterprise. Suppliers, OEMs, and other individuals involved externally need a form of the product that does not contain proprietary information or excess data that they do not need, let alone inaccurate data that may have been corrupted during translation. Even inside of the MBE, limiting the amount of information present at specific stages of the lifecycle can increase productivity and reduce time spent sifting through excess information [6]. Identifying the minimum information model needed to effectively replace a drawing also provides standards development organizations (SDOs), corporate PLM practitioners, and the PLM software community a more stable target when developing guidelines and policy for creating, translating, and validating model-based definitions. Being able to identify the minimum information model, and where it sits in the lifecycle, can help companies adopting MBD develop an understanding of how this important information can be captured and represented. The MIM can also help to develop validation and adoption strategies for technology used for modeling, translating, and validating data.

The research outcomes presented in this report were driven by the need to understand what information items constitutes the minimum information model and why the MIM is important to the authors and consumers of information in the selected workflows (concept to prototype, prototype to detailed product definition, detailed product definition to manufacturing, and manufacturing to inspection). While the workflows examined in this study do not represent an exhaustive list of possibilities, they do represent interactions between people that account for a great deal of the authoring and initial consumption of digital product data.

\section{Background Literature Review}

Model-based Definition is emerging in industry as a communicative platform that is replacing the use of technical drawings, especially in companies that have the capacity to identify and execute on the information needs of an MBD or model-based enterprise. A modelbased definition provides a unique view of product information, and has the potential to enable the consolidation and representation of implicit and explicit. The information elements provided using an MBD are potentially many, and the creation of a digital model can allow the company to leverage that information in a more powerful communicative fashion than provided by drawings historically. Many industries, the Aerospace and Automotive industries especially [1], are leading the adoption of MBD in the transition from drawings to models. 
Being able to utilize the information provided by the creation of an MBD reduces the need for 2D drawings, especially as the concept of a document of record becomes increasingly focused on the 3D model and its associated data [7]. Eliminating the use of 2D drawings or changing them from the master definition to a reference document is slowly becoming the norm being seen in industry [8]. Drawings were historically used to communicate information before model-based definition or the development of these 3D CAD systems. In the manufacturing or production environment, they contained instructions on how to create products within the respective design space with the tools available. Drawings contained a combination of implicit and explicit information, much of which was tied to the contextual environment where the drawing was used. The information on the drawings, therefore, became more about the product definition rather than simply capturing the geometric shape [9].

This increase in complexity of the drawings meant that the user or consumer of these drawings would need greater understanding of the context and the semantics of the drawingbased product and process information in the specific workflow in which they were used [10]. A gap exists between companies based on their individual interpretation and representation of drawing standards used in the creation of their drawings. When left open to interpretation, there are a variety of ways to apply these standards, and even more ways to interpret them. Implicitly defined information is often some of the most critical information found on a drawing, with many design, production, inspection, and support tasks dependent on it. The gap that emerges gets larger when the information needs to go outside of the company, or when it is disseminated throughout the supply chain. Moreover, the interpretations of drawings from one industry sector to another differ so drastically that different actors could receive the same drawing and interpret it differently [5]. Even users that participate in different workflows, but utilize the same drawing, will come away with their own interpretation [11].

The emergence of MBD data has not necessarily solved these issues, because the differences in interpretation remain a prevalent problem in industry. One problem that affects the digitalization of the product definition is the increasing of space between organizational functions, very similar to the way it has affected the use of drawings. Historically, if there were questions about the information on a drawing or with respect to a process, the user could simply go back to engineering or to the design team and have their questions answered. Today, not all functions in a company (engineering, design, production, manufacturing, sales, etc.) exist in the same geographic location, and rarely do people communicate between one another on a personal level that is accessible to the larger organization. Informal communication that formerly existed when drawings were the master definition is being replaced by explicitly defined information in an increasingly digital environment. The different forms of the MBD are now required to be explicitly defined to be shared accurately among a network of global designers, suppliers, and service personnel $[12,13]$. Large companies tend to operate in multiple, global divisions today. Networks of suppliers, OEMs, consumers and $3^{\text {rd }}$ party management, now more than ever, demand that information be represented in a way that isn't confusing and that can be translated effectively. While these problems did exist with drawings, the ability for informal communication to take place quickly, and the use of a communications medium (i.e., paper) that 
did not change frequently, allowed for effective mitigation of interpretation error (only if the employee actively sought guidance instead of utilizing their own interpretation, however).

While digital architecture becomes a growing force in industry as companies move toward digital MBD representations over 2D paper-based representations, the sharing of information has been made easier by the digitalization of formerly physical communications assets (i.e., drawings). However, it has increased the difficulty of gathering all the necessary information into one place. With drawings, information such as materials, work instructions, etc. could easily be included on the physical drawing by reducing the information to textual or symbolic, hand-written forms, and the paper (or mylar, vellum, etc.) served as a common and stable dissemination platform. With digital 3D representations, a challenge exists with getting the necessary information into the product definition in a way that is accessible by everyone who touches the product and uses the digital model artifact. In addition to the interpretation of the information the distribution of information is just as imperative [14], especially when the product definition needs to be shared over several different workflows or across several industries.

\section{Research Study Overview}

A staged study approach was used to identify required information elements. We studied the workflow identification and element identification first, then generated IDEF0 diagrams of the workflow activities. Stage One of the research identified workflows that are common across industry sectors and their basic information needs and usage. The four workflows established in Stage 1 were:

- Concept to Prototype

- Prototype to Detailed Product Definition

- Detailed Product Definition to Manufacturing, and

- Manufacturing to Inspection.

Each workflow has separate elements associated with it, defined either in the supporting documentation or through interviews with practicing industry professionals involved in similar workflows. Industrial efforts on modeling workflows for the global design process proved invaluable when understanding the use of technicians, design teams and order of operations for a prototyping workflow [15].

The element identification portion of the study utilized a three-round Delphi study with two supplementary interview rounds. In Stage Two, this research study sought to establish the relative significance of the MIM elements associated with it and the common information model.

Stage Three of the study used Integration Definition for Function Modeling (IDEF) modeling techniques to capture the inputs, outputs, constraints, and tools used in the selected workflows. One round of interviews was used to contextualize the results of the IDEF0 models. IDEF is used to describe the modeling language of IDEF0 along with the associated rules, techniques and semantics. Level 0 of the IDEF modeling structure is used to formulate graphical 
representations of a system or an enterprise network through relationships, functions or data that supports systems integration [16]. It is also hierarchical in nature, consisting of diagrams, text or glossaries that all reference each other [17]. In this study, IDEF0 charts were created based on workflows identified in Stage One to show graphically how the data interacts with itself throughout the system. In association with NIST, the IDEF0 diagrams were created from research done on the ISA-95 Part 4 manufacturing environment standard, in conjunction with ANSI/ISA-95.00.03-2005 [18-21]. The literature and standards portrayed interfacing content between manufacturing operation environments and management environments. The sample diagrams provided within the ISA-95 framework laid the groundwork for high-level views of the four workflows. The IDEF0 charts were created in Microsoft PowerPoint as a proof of concept, but were later imported to a more interactive environment, Innoslate ${ }^{1}$, which leverages webbased tools to allow users to generate IDEF0 diagrams. Similarly, work done to model the flow of data throughout multiple workflows influenced the layout of the IDEF0 diagrams created here. Once the initial IDEF0s were created, interviews took place to discuss the order and flow of information. Changes were color-coded to match the respondents' suggestions and corrections as well as to highlight CIM/MIM information present in each workflow. This report presents the findings from all three stages and attempts to identify and characterize the minimum information model in the selected workflows to illuminate the information needs across the lifecycle stages.

\section{Stage One: Identifying Workflows and Demographics}

The first stage of the study targeted industry professionals with the goal of identifying primary information in various workflows to help establish an understanding of the common information model and minimum information model as they pertain to the four targeted workflows. Figure 1 shows the spread of industries that participated in the study. Of the 76 respondents, $85 \%$ of them were located inside of the U.S. with $15 \%$ located outside.

\footnotetext{
${ }^{1}$ https://www.innoslate.com/
} 


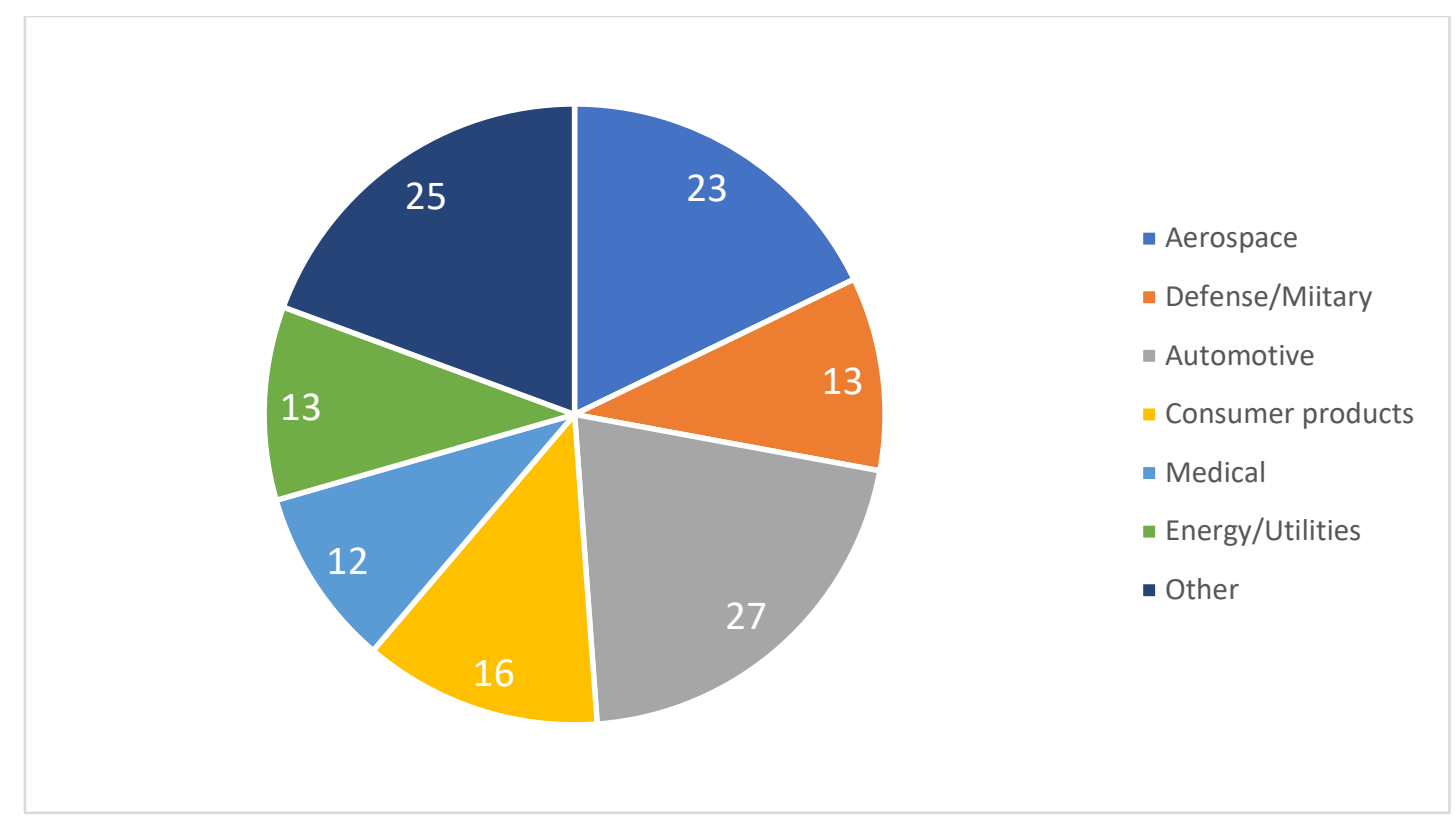

Figure 1: Distribution of Industry Participation (N: 76)

We collected demographic data on the survey participants. The first couple of questions ask questions about the company with which the participant works currently, with the participants identifying company size, location and industry setting. The selections for size of company varied by 50 employees each, and capped at "500 or more". They were then asked to identify their job area within the company, with a text box entry allowing for participants with areas not on the identified list to type in their job area.

The next set of questions asked the participants how they receive engineering information and in which workflow they most actively participate. The participants could choose only one of the four targeted workflows, but could also specify that they did not participate in any of the workflows shown. If they did not participate in the workflows, then the survey immediately ended and their participation was concluded. While this approach can be debated, the research team decided to use the four identified workflows as an initial boundary mechanism for this study.

Depending on the workflow selected, the survey followed one of four branches with similar questions, with the first branch asking about what type of information was created and used in their self-selected workflow. A list of possible information item types was provided to the participants from which to choose, as well as a free-response field for them to provide their own answer. Next, they were asked to identify if drawings were used as the medium to communicate the information items they selected. If they selected no, they were prompted to answer what other documents were used to communicate besides drawings. To gain an understanding of the respondents' use of 3D data, they were asked if the information items selected previously could be communicated inside of a 3D model. If not, the participants were asked to identify why the information could not be communicated inside of a 3D model in their 
workflow was presented. However, if yes was selected, a final question asked the participants to provide any comments they had on the topic of annotated 3D models replacing drawings.

The structure of the Stage One survey was important for gathering specific information about each workflow. It built an understanding of the participants’ knowledge of MBD, especially as it related to their job role and industry sector. Specific results of the survey are shown in Figure 2 through Figure 5 as they relate to the use of 3D models and some of the challenges associated with using models as a replacement for drawings.

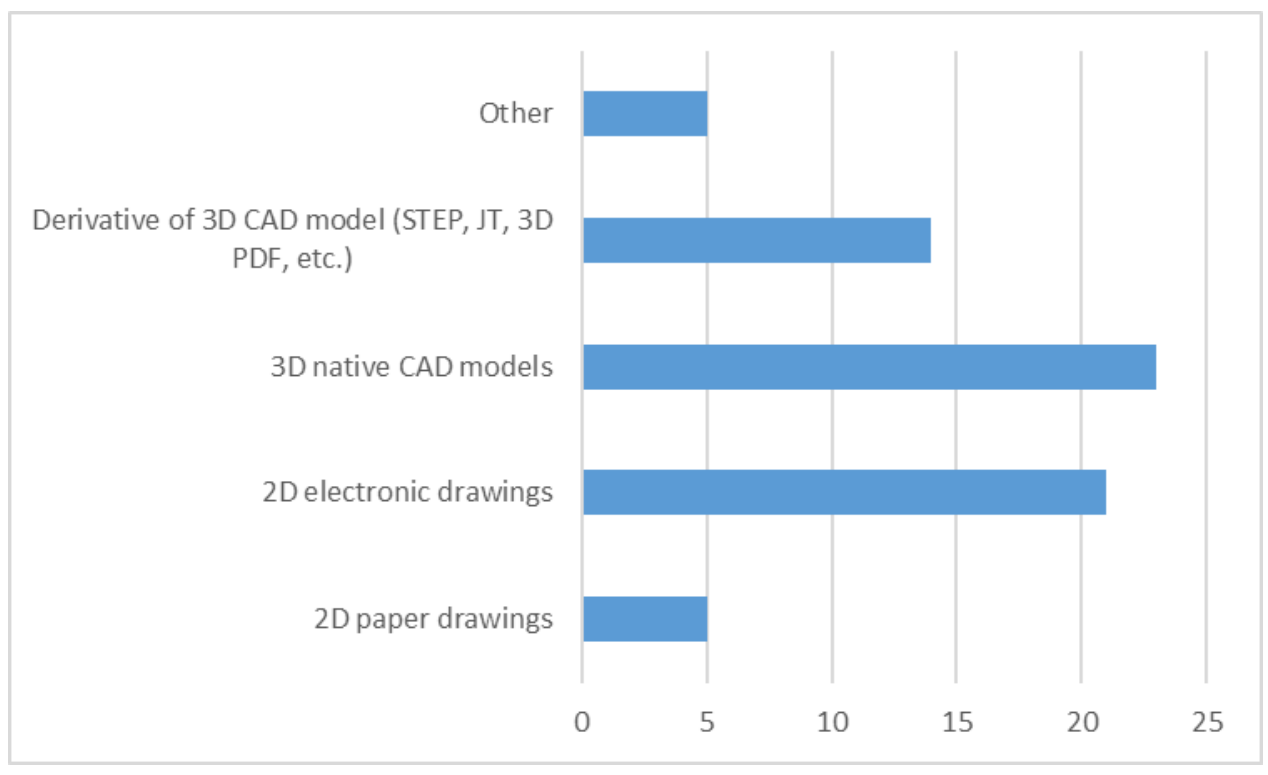

Figure 2: How participants receive information (N: 68)

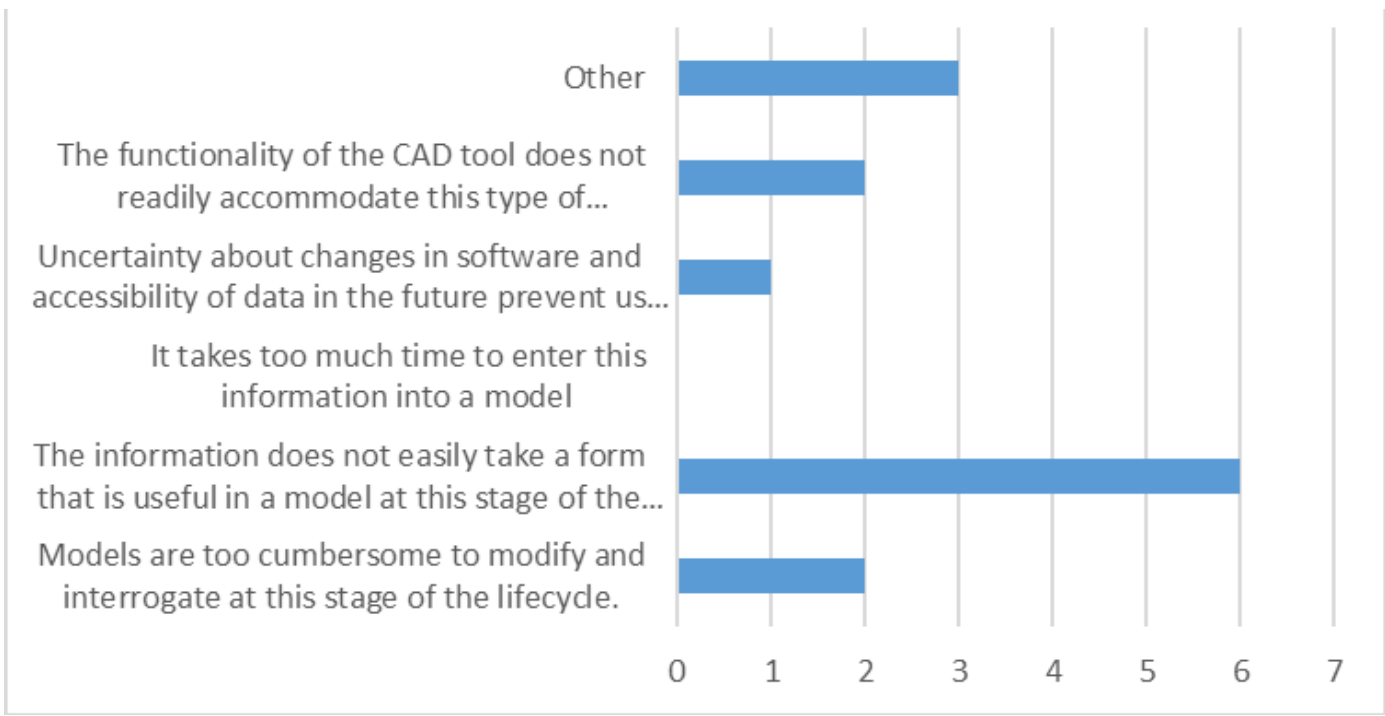

Figure 3: Issues that make adoption of MBD challenging (N: 14) 


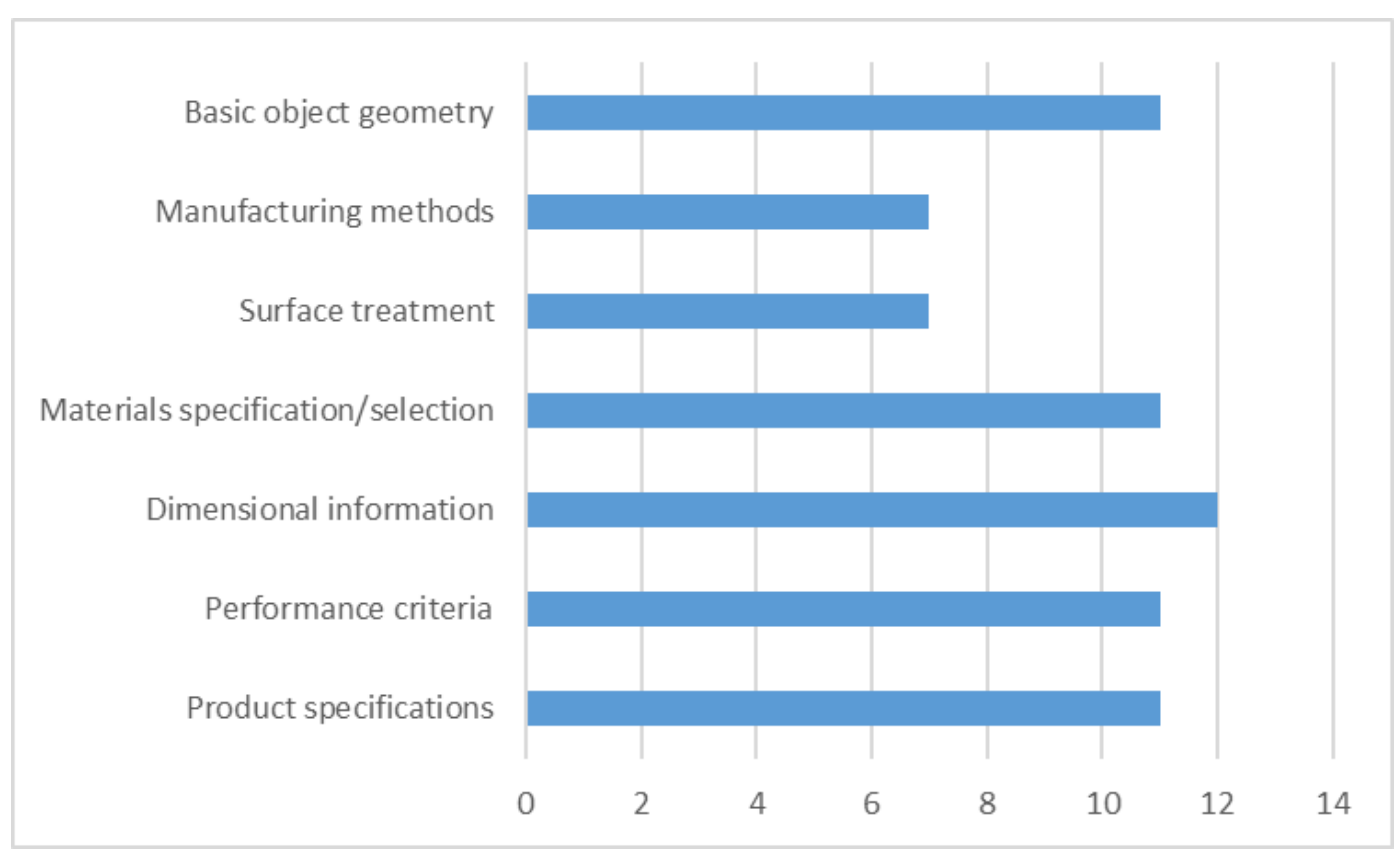

Figure 4: What information was created or used in the product information for the Concept to Prototype workflow (N: 70)

\section{Could Models be Used in Place of Drawings?}

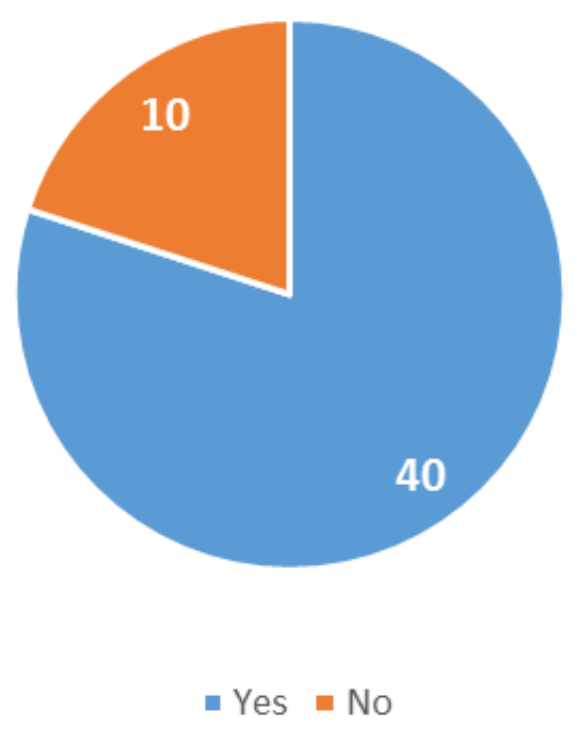

Figure 5: Could models be used in place of drawings in your workflow? (N: 50) 
The first stage resulted in the discovery of a main issue of adoption, information does not take on a form that is easily incorporated into a model or even particularly useful to the model inside of the workflows identified, as illustrated by Figure 3. Having a complete understanding of the information that flows inside of an organization is key towards adopting a model-based definition or model-based enterprise. A large portion of that information is divided into workflows, and understanding what information goes inside of each workflow is what the minimum information model seeks to understand. The information being heavily referenced here and in the next paragraph refers to the "data" or "information" that is generated and shared within a company about a specific product or process. When designing a product, companies don't always start from scratch. Old, stored information, can be referenced or re-used to provide a start to a new product. Drawings have implicit and explicit information, discussed earlier, but word of mouth is also another transfer of information. Gathering all the "data" associated with a product can be extremely difficult, but then getting that data into a form that can be applied to MBD is even more strenuous.

Capturing implicit information that was formerly used in drawings to be represented in MBD is the first step towards complete adoption. A main issue, results from the first stage, speaks about the data fidelity and compatibility across multiple systems. Model-based definition allows users to digitalize formerly physical data, but when that data is shared across multiple tools, CAD software or corporations, the fidelity can become skewed and information can be lost. By understanding what information goes into the workflows associated with a product or lifecycle, the common information between workflows becomes clear and domain-specific information can begin to be identified. Stage 1 of the study exposed information being consumed by the respondents within the four identified workflows, but does not reflect all information inside each workflow. The next two stages in the study focused heavily on understanding the information inside of the workflows, as well as how that information flows from one workflow to the next. Stage Two used a Delphi study technique to identify the information elements associated with each workflow, and to understand their relative importance to each other and to the larger set of workflows in the study.

\section{Stage Two: Establishing the Importance of the MBD Information Items Using a Delphi Study}

The Delphi technique [22] was used to identify the elements needing to persist in a model-based definition throughout the lifecycle. Overall, the Stage Two performed three separate rounds of questions to identify and assess the information collected, and to establish which elements are truly necessary for all stages of the lifecycle. The common information model is evaluated alongside the minimum information model throughout the three rounds of Stage Two. The minimum information model identifies what information is necessary inside of the workflows whereas the common information model identifies the elements that persist throughout all workflows in the lifecycle. IDEF0 diagrams were used by the Delphi study to 
visualize the workflows identified and display where the elements evaluated fit into those workflows. The IDEF0s were subject to interviews as well to verify the information presented in them.

\subsection{Delphi Survey Round One}

The first round of the Delphi study utilized twenty-two separate information elements identified by reviewing the relevant domain literature and from participant responses and comments in Stage One. While there is great interest in understanding the concepts of modelbased enterprise (MBE) and model-based definition (MBD), and how they can be used to improve the information flow and handling of data throughout the lifecycle of a product, there is much uncertainty and inconsistency in the manufacturing community about the information items to be included in an MBD and how that MBD should be used in lieu of a drawing. Round 1 of the Delphi process was conducted to allow participants to provide input on how often they use specific information elements and which elements are more important to them. It was expected based on the workflows they chose, that the responses would vary widely, and perhaps even provide insight into new information elements or reduce the list of elements currently provided.

After gathering information about what elements were necessary inside of a participant's specific workflow, the survey asked which elements are necessary based on the participant's identified workflow, which elements are common throughout the lifecycle, and to what extent the participants used drawings or models to complete their jobs. Figure 6 and Figure 7 show the responses to each of these questions, the obvious outcome being that the geometry definition and information related directly to it are the most common information elements from one stage of a lifecycle to the next, and the most necessary for communication inside of workflows.

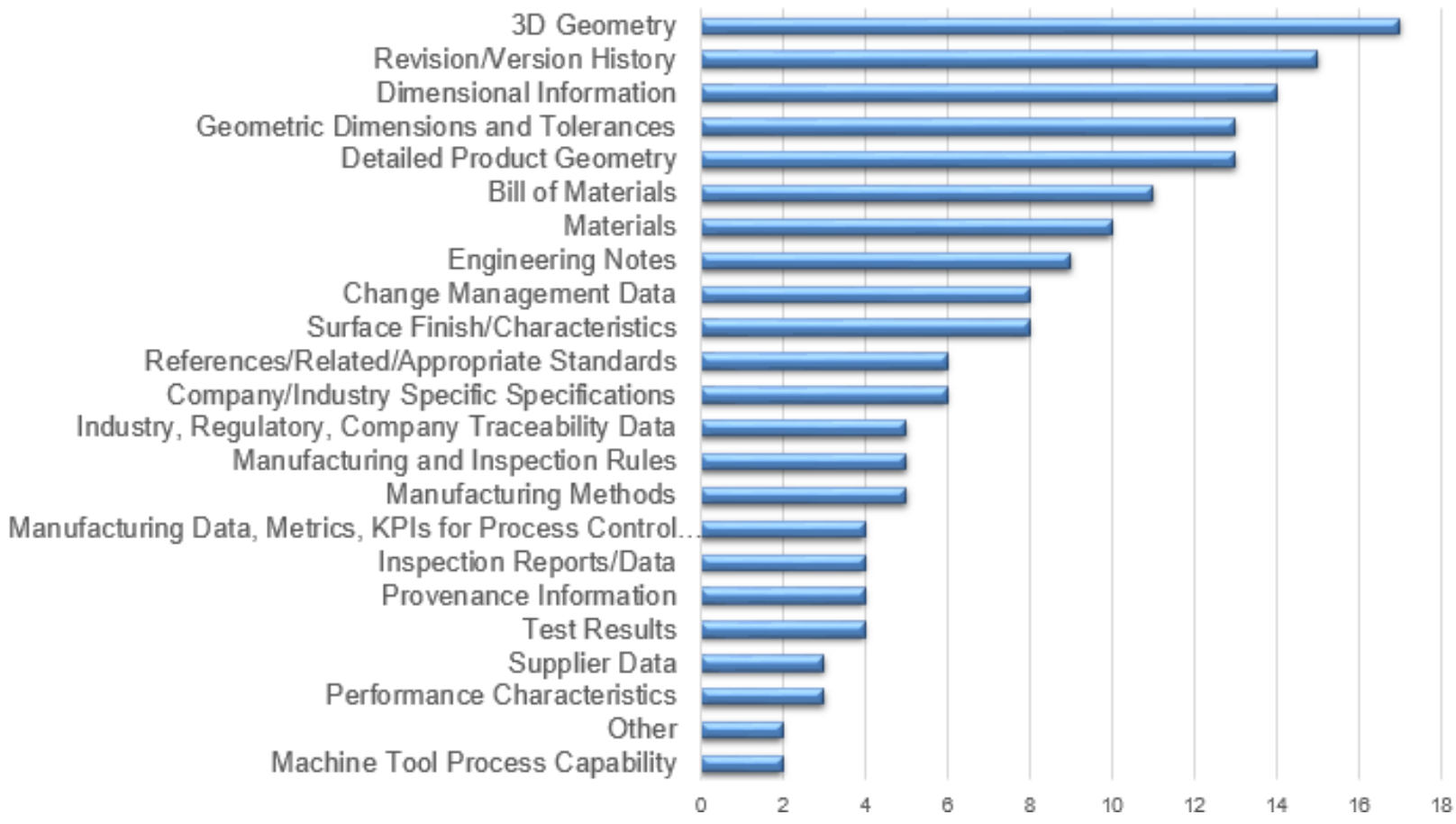


Figure 6: Assuming you use a MBD across the lifecycle of your products, which elements are common from one life cycle stage to the next? (Select all that apply) N:46

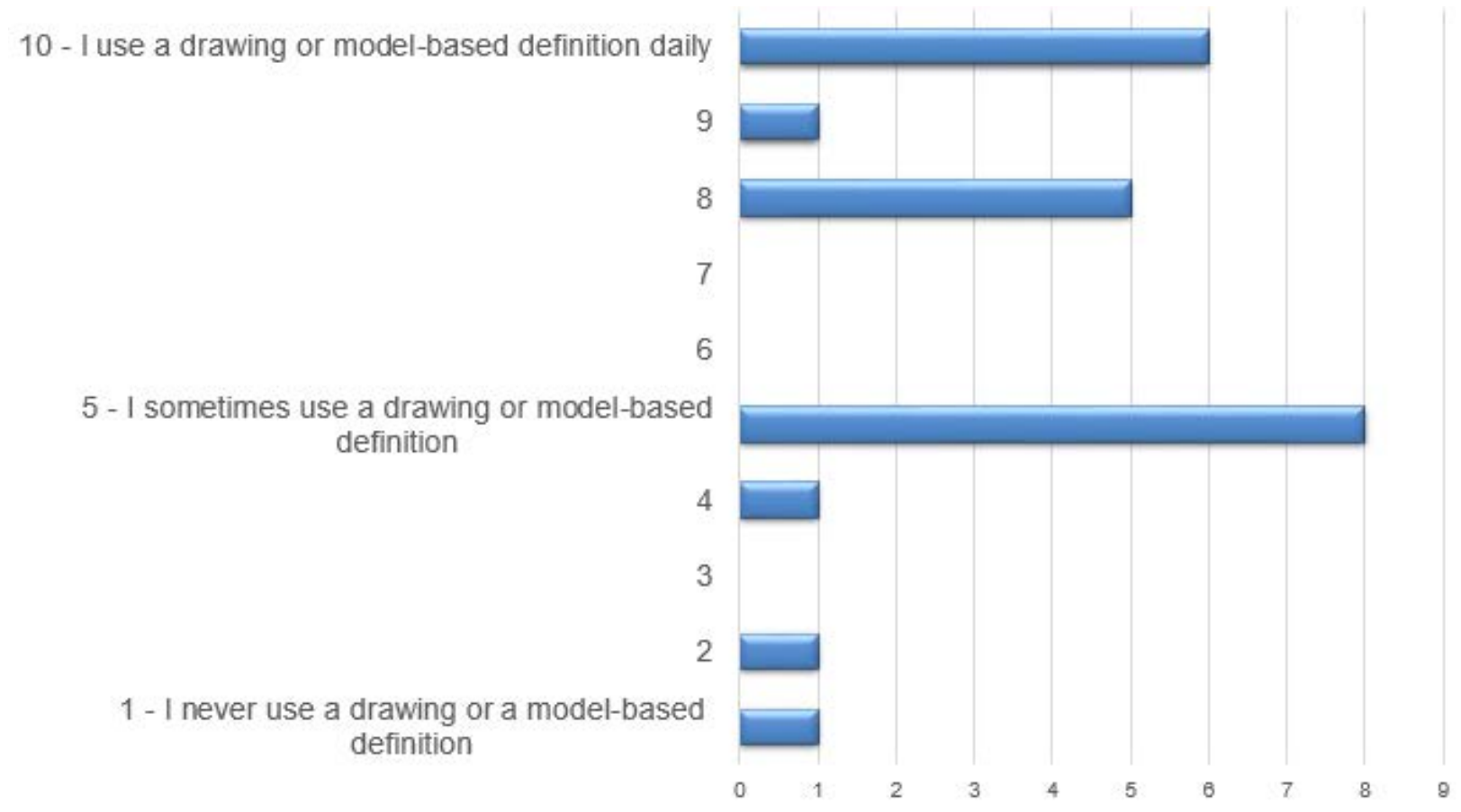

Figure 7: To what extent does your job role involve the use of a drawing or model to complete your job effectively? N: 23

Round 1 Delphi data tells us that the use of model-based definitions has begun to make its way into the daily work of the survey respondents. And to the extent that the survey respondents are representative of their industry sector, it is fair to say that the use of MBD is impacting aerospace, automotive, and defense/military more than other sectors currently. It is also fair to say that, while MBD usage is beginning to become common across the industry sectors, the use of drawings is still particularly high as well. The nature of this first round was to gather a broad view of the industrial sectors that can be sampled from and to gain an understanding of the penetration of MBD in across those sectors. The information elements identified provided a broad scope for the initial understanding of how model-based information is used in industry. Round 1 also provided an opportunity for respondents to provide additional input or information categories, which the author team may have missed. This is extremely important when utilizing a Delphi method, as the subsequent rounds are to be more succinct and critical in their analyses.

Delphi Round 1 was driven by questions seeking to understand if the participants were authors or consumers of MBD or not, if they were considered OEMs or suppliers, and other contextual questions to determine the level of importance they placed on the identified information elements. In industry, there is a lack of common consensus on the definition of MBD and how it can be used to streamline information sharing in a specific workflow or how it can improve the quality of information produced and shared. The participants in Delphi Round 1 represented varying industry sectors, as seen in Figure 8. Delphi Round 2 (Figure 10) and Round 
3 (Figure 12) show a diverse mix of industry sectors as well. The diverse mix of responses allowed for a consensus to be reached on certain questions, but did not inhibit the separation of answers based on industry. For example, Figure 8 shows the responses from eight different industry sectors, as well as an "other" option. Of the 23 responses, five came from the Aerospace industry, and most of those responses were a 10 or an 8 on the scale of one-to-ten. Of the next category down, Defense and Military, the responses ranged from a two all the way up to a ten. This varying trend provided insight into the industry sectors which might be more advanced in terms of MBD creation and usage versus those industries that are still in the very early adoption phase, or perhaps those which are not even attempting to adopt MBD.

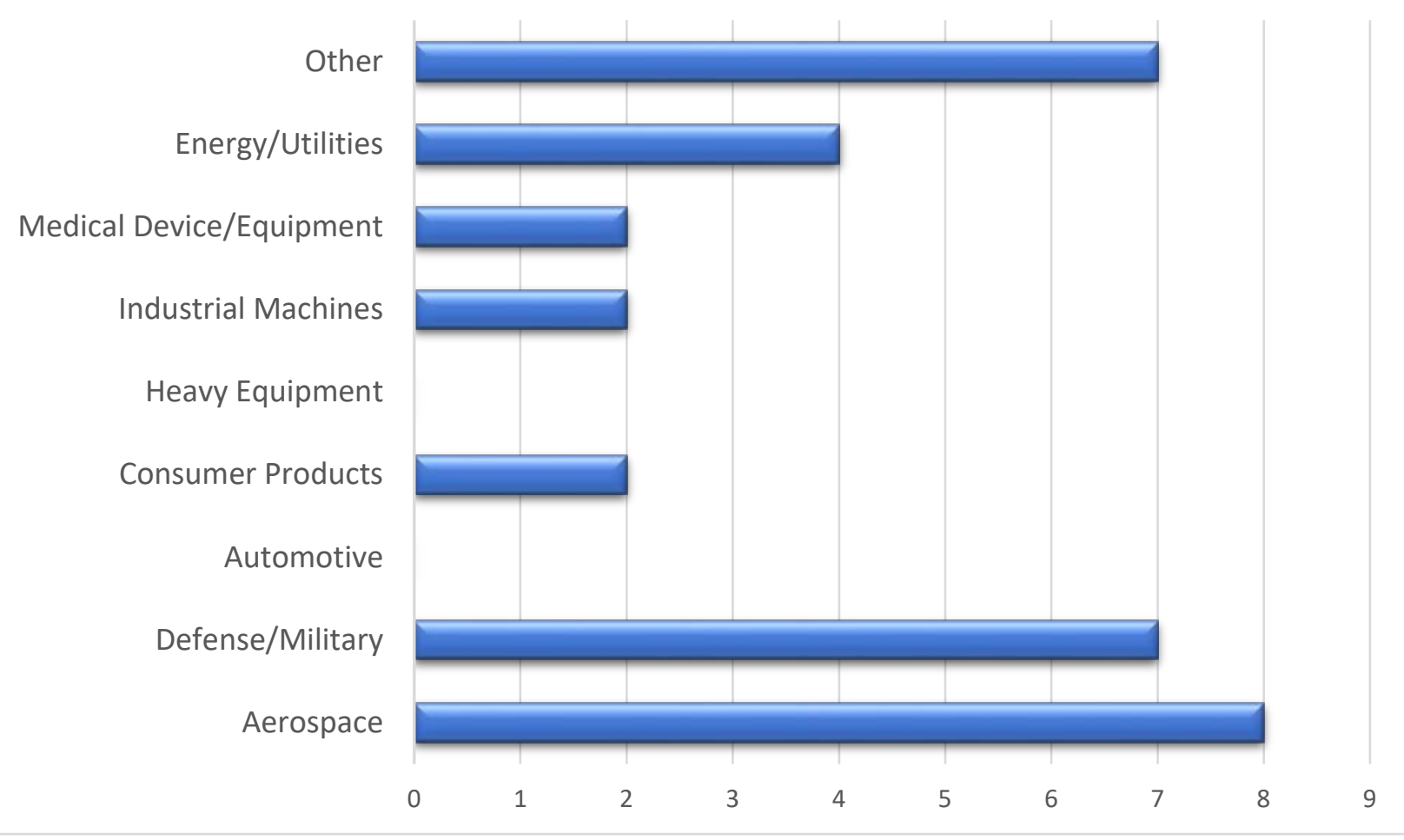

Figure 8: Which industry sector best represents your company, or the division of the company where you work? (N: 32)

There is much focus on the effective and accurate creation, translation, and validation of digital product data across the lifecycle, but there has been little (if any) work done to date on which information elements are important or why and to whom their importance matters. The Delphi survey Round 1 allowed for the collection of MBD potential usage data and its importance to the respondents based on their industry, workflows and personal opinions about the data in question. Round 2 of the Delphi study (described in the next section) asked the survey participants to rate the relative importance of each information element from Round 1 according to the participant's workflow and job role to reach consensus on the importance of each information element. 


\subsection{Delphi Survey Round Two}

The Delphi survey Round 2 focused solely on the MBD information elements identified from Round 1. The participants were asked to rate the elements on a scale of one to seven, with one being "not critical” and seven being "most critical". The first half of the survey asked the participants to rate the importance of including each of the twenty-two information elements in an MBD used in the participant's specific workflow. The second half of the survey asked participants to rate those same information elements on a scale of importance for being present, regardless of the participant's specific workflow. The second set of questions (i.e., importance of the information independent of the participant's specific workflow) encouraged the participants to think broadly to gain a basic view of the importance of a participant's specific workflow and job role on their perception of the importance of a given information item. An example of the survey questions is shown in Figure 9, and the entire survey is included in the appendix. 
Q4-26. The following questions will be used to identify the most critical pieces of information that should be included in a model-based definition for a given workflow. At minimum, which of the following should be included in the MBD for the workflow you participate in? Please rank each item (1 - not critical, 7 - most critical).

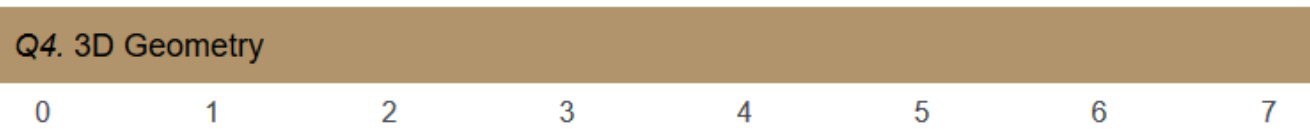

\section{Q5. Dimensional Information}

$\begin{array}{llllllll}0 & 1 & 2 & 3 & 4 & 5 & 6 & 7\end{array}$

\section{Q6. Detailed Product Geometry}

0

1

2

3

4

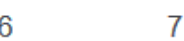

\section{Q7. Revision/Version History}

0

1

2

3

6

7

\section{Q8. Geometric Dimensions and Tolerances (GD\&T)}

$\begin{array}{lllllllll}0 & 1 & 2 & 3 & 4 & 5 & 6 & 7\end{array}$

\section{Q9. Materials Specifications/Definitions}

$\begin{array}{llllllll}0 & 1 & 2 & 3 & 4 & 5 & 6 & 7\end{array}$

Figure 9: Example of Delphi survey Round 2 
After finalizing the results from the Delphi survey Round 1 and formulating the questions for Delphi survey Round 2, it became apparent that elements of a MBD do not share common meaning between people based on industry sector, job role, and use of these elements across the lifecycle. Sample results of Delphi survey Round 2 are shown in Table 1 and Table 2. After collecting the results and analyzing the 42 responses acquired, follow-up interviews with volunteers from various industry sectors to confirm the necessity of the elements that had the highest response rates. Over the course of twelve interviews with industry professionals (the results of which are discussed in the next section), the contextual responses provided a framework for to understand the statistical analysis of Delphi survey Round 2 responses.

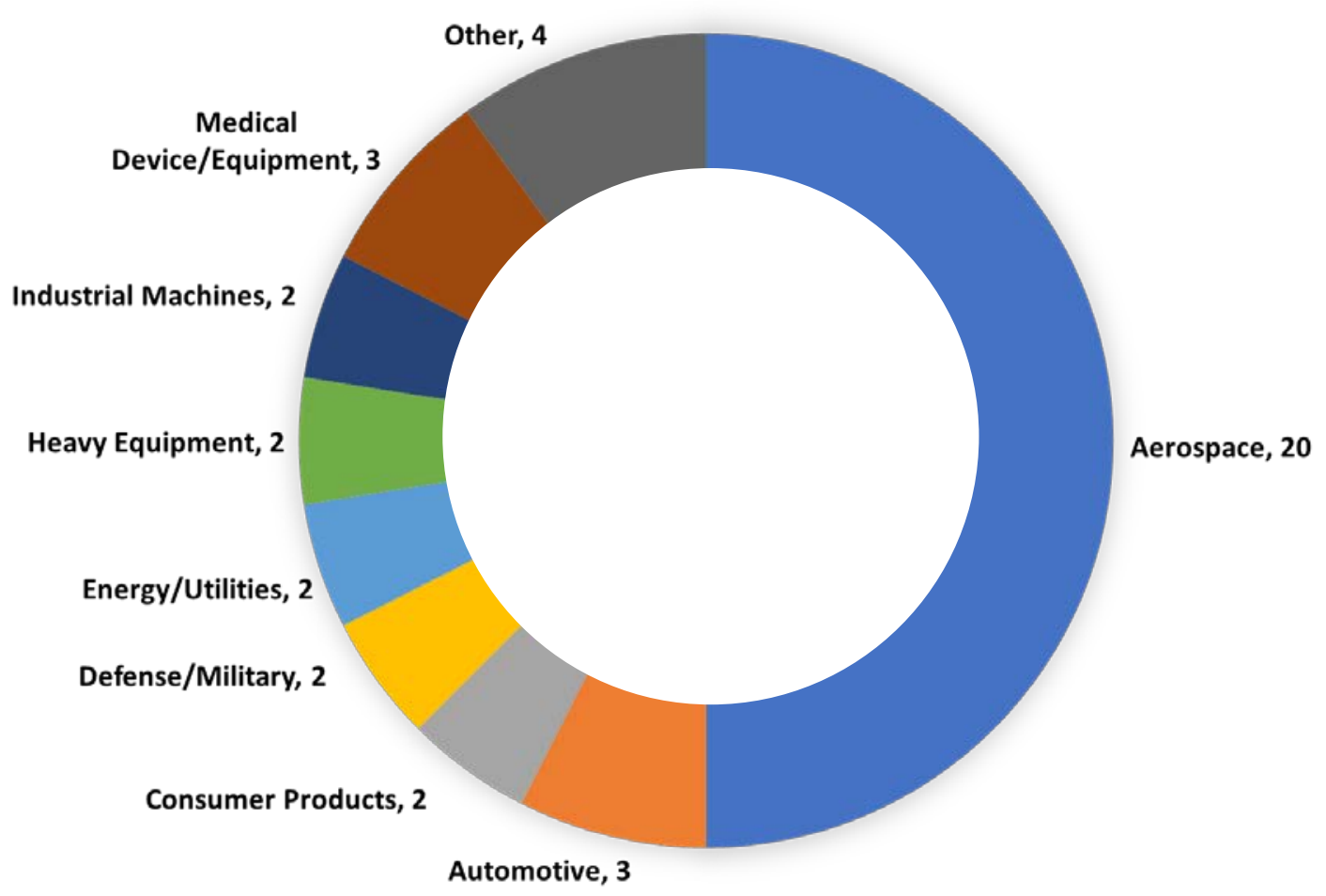

Figure 10: Q1 - Which industry sector best represents your company or the division of the company where you work? 
Table 1: Questions 4-9 Delphi Study Round Two

\begin{tabular}{|c|c|c|c|c|c|c|}
\hline Question & $\begin{array}{c}\text { Minimu } \\
\mathbf{m}\end{array}$ & $\begin{array}{c}\text { Maximu } \\
\mathbf{m}\end{array}$ & Mean & $\begin{array}{c}\text { Std. } \\
\text { Deviatio } \\
\mathbf{n}\end{array}$ & $\begin{array}{c}\text { Varianc } \\
\text { e }\end{array}$ & Count \\
\hline 3D Geometry & 3 & 7 & 6.65 & 0.82 & 0.68 & 40 \\
\hline Dimensional Info. & 1 & 7 & 5.85 & 1.56 & 2.44 & 39 \\
\hline $\begin{array}{c}\text { Detailed Product } \\
\text { Geometry }\end{array}$ & 2 & 7 & 6.21 & 1.24 & 1.55 & 39 \\
\hline Revision/Version History & 2 & 7 & 5.58 & 1.44 & 2.09 & 38 \\
\hline $\begin{array}{c}\text { Geometric Dimensions } \\
\text { and Tolerances }\end{array}$ & 1 & 7 & 6.03 & 1.37 & 1.87 & 39 \\
\hline $\begin{array}{c}\text { Materials } \\
\text { Specifications/Definition }\end{array}$ & 1 & 7 & 5.66 & 1.72 & 2.96 & 38 \\
\hline s & & & & & & \\
\hline
\end{tabular}

Table 2: Questions 27-32 of Delphi study round two

\begin{tabular}{|c|c|c|c|c|c|c|}
\hline Question & $\begin{array}{c}\text { Minimu } \\
\mathbf{m}\end{array}$ & $\begin{array}{c}\text { Maximu } \\
\mathbf{m}\end{array}$ & Mean & $\begin{array}{c}\text { Std. } \\
\text { Deviatio } \\
\mathbf{n}\end{array}$ & $\begin{array}{c}\text { Varianc } \\
\text { e }\end{array}$ & Count \\
\hline 3D Geometry & 3 & 7 & 6.41 & 1.08 & 1.16 & 37 \\
\hline Dimensional Info. & 2 & 7 & 5.64 & 1.58 & 2.51 & 36 \\
\hline $\begin{array}{c}\text { Detailed Product } \\
\text { Geometry }\end{array}$ & 5 & 7 & 6.06 & 1.13 & 1.27 & 36 \\
\hline Revision/Version History & 1 & 7 & 5.75 & 1.61 & 2.58 & 36 \\
\hline $\begin{array}{c}\text { Geometric Dimensions } \\
\text { and Tolerances }\end{array}$ & 0 & 7 & 5.58 & 1.72 & 2.97 & 36 \\
\hline $\begin{array}{c}\text { Materials } \\
\text { Specifications/Definition }\end{array}$ & 1 & 7 & 5.14 & 1.80 & 3.23 & 36 \\
\hline $\mathbf{s}$ & & & & & & \\
\hline
\end{tabular}

A key difference between Tables 1 and 2 is that while the questions are asking about the same information elements, they are asking about those elements in different contexts. Table 1 shows an excerpt of the questions about the information elements according to the participant's own workflow, while Table 2 asks about the importance of the same information elements independent of the workflow the respondents participate in. The results shown in Table 1 show that 3D Geometry (Q4 and Q27) is very important in both the respondent's workflow and independent of workflow. To better visualize where the importance of each information item was relative to the average response score for each item, an ANOVA was conducted, with the results plotted on the chart shown in Figure 11. 


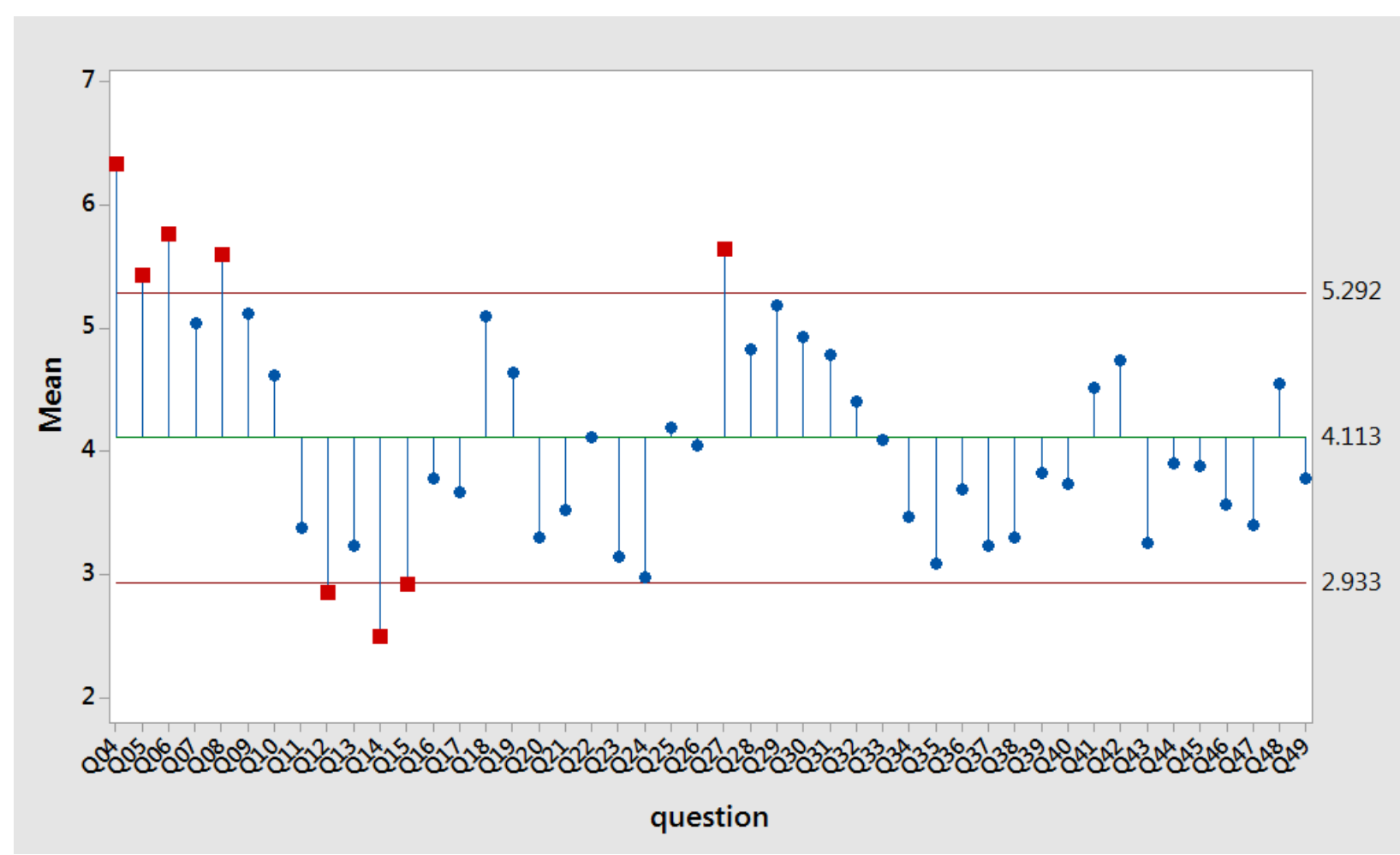

Figure 11: ANOVA data for Delphi survey Round 2

The upper and lower standard deviation limits for the various questions are the two horizontal lines seen at the top and bottom of Figure 11. The green horizontal line (directly through the middle) shows the average rating for the information elements. Any information element with an average rating below the mean were eliminated (e.g., on a rating scale of $1-7$, a value of 4 represents the neutral mark). Any information elements whose average rating was on the mean or extremely close to the mean were scrutinized further to decide if those elements had been accepted in one case and denied in another, or universally denied or accepted. The result of this analysis was that twelve information elements were retained for discussion in the interviews and for Delphi survey Round 3. The interviews following Delphi survey Round 2 verified that the elements chosen to remain were of value to industry.

\subsection{Delphi Survey Round Two Interview Data}

In total, nine industry professionals were interviewed as a means of providing context to the responses in Delphi survey Round 2. Several factors emerged from the interviews, which had an impact on the decision to conduct Delphi survey Round 3. The first important factor was the discussion of MBD tools and methods and how easily (or not) they can be adopted. According to the survey in Stage One of this study, model-based definition methods are often difficult to learn and implement, and MBD tools can be difficult to use. The steep learning curve that is presented by the software; the cost and instability of the computing infrastructure; and the information authoring and consumption methods utilized in an MBE environment create a barrier for adoption of MBE and dissemination of information. These challenges have been further exacerbated by the growing chasm between users who are well versed in the methods and tools 
for creating 2D drawings and those who have been using 3D modeling tools for their entire career.

The interviews following Delphi survey Round 2 exposed an opinion that training someone to utilize MBD tools and methods can be quite challenging, as it requires proper infrastructure, data model creation and exchange, and a great deal of time. MBD also requires someone to know and understand the product lifecycles within the company, the various workflows and subdivisions involved in those lifecycles, and how digital product data flows between and supports the product lifecycle. Without proper training, and corporate process and practice to support and reinforce the training, MBD adoption and utilization will likely be flawed and unsuccessful. According to the interview participants, some companies have had a relatively smooth transition to the use of MBD tools and methods by utilizing a hybrid approach involving the use of a drawing in addition to the model.

The Delphi survey Round 2 interviews also suggested the culture of a company will have a major impact on the adoption of any new MBD process, technology or architecture. Larger companies will move slower than smaller or new companies, simply due to their size and the amount of effort that goes into converting from drawings to models (cost, time, training, etc.). The effort to find information, to clean product data for conversion, and the product data compliance and traceability issues in a larger company are also conditions that can be challenging for MBD implementation. Likewise, the culture of a company can rapidly increase the adoption rate of any new MBD technology or process. Companies that drive change and are early adopters emerging technology will move very quickly compared to those that have iterative upgrade procedures, rigorous rules on the adoption of technology, or limitations based on budget or personnel. Looking at the individual, those who have experience working with large amounts of technology should adapt to change quicker, especially in the utilization of a new technology to increase work efficiency.

Interviewees also noted the disparity in newer employees versus those who had been with the company for a majority of their career. In many companies, experience and seniority are emphasized when selecting personnel for leadership positions. However, many of these people today lack the experience and affinity with technology that younger (and arguably less experienced) employees have. However, it is usually those younger employees that are most fluent with and accepting of MBD tools and methods. College graduates emerging into the market come bearing a unique set of skills and knowledge of technologies either practiced personally or studied in school. However, upon reaching full-time employment, there is often a culture clash as these individuals are immersed in older methods and taught to utilize dated technologies. The presence of fresh talent should encourage change and speed up the adoption of new technologies and processes, especially those presented by MBD or MBE. As new talent continues to circulate into the market, and those with all the experience start to circulate out, a new understanding of technology in those companies will emerge, combined with experience shared by mentors and peers.

The face validity provided by the interviews prior to Delphi survey Round 3 helped narrow the questions that would be asked, and presented some new questions used for probing 
about culture, education, and adoption in the third round. The interviews also further set the context for verifying the relative significance of the twelve information items from Delphi survey Round 2. This next section will discuss Delphi survey Round 3 and the results that were established from the survey relative to the industry viewpoints on MBD/MBE adoption, as well as similarities and differences between the twelve information elements.

\subsection{Delphi Survey Round Three}

Understanding how individuals think about the concept of model-based definition, and the data that goes inside of it, has been the primary focus of this study. Delphi survey Round 3 used the resulting elements from Round 2 and surveyed industry participants like the way Round 2 was conducted. Delphi survey Round 3 was used to verify the importance of the refined list of information elements gleaned from the two previous survey rounds, and to gain an understanding of how industry currently views model-based definition as well as model-based enterprise. Participants in Round 3 were asked to rate the twelve information elements presented on a scale of importance from one to seven, with one being "not important" and seven being "very important”. Fifty-three participants provided responses to the Delphi Round 3 survey, with ratings that verified the information elements found in previous rounds. A desired outcome of Round 3 was to understand the similarities and differences between the common information elements.

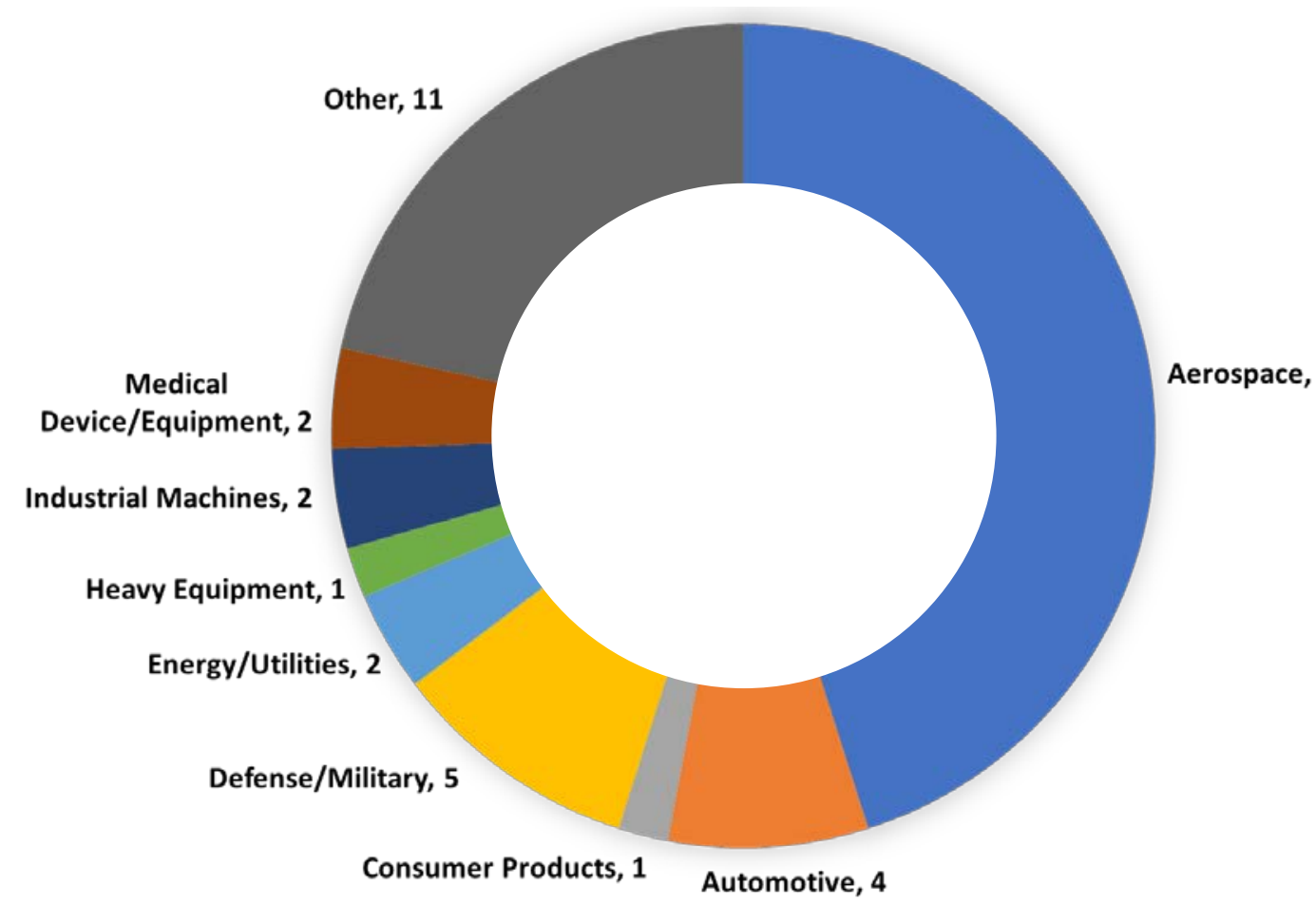

Figure 12: Q2 - Which industry sector best represents your company or the division of the company where you work? (N: 51) 
Figure 12 shows the distribution of industry responses for Delphi survey Round 3. Just like previous rounds, the Aerospace sector had the most representation. Some of the "other" descriptions of industry participation were "PLM Software, Consulting, OEM mfg., Aerospace and Defense, Software services and Computer, Servers, Software”. Most of the survey results came from Q6 through Q17, which asked the participants to rate the twelve information elements on a scale of importance from one to seven. Figure 13, Figure 14 and Figure 15 below show the results of the ratings. One important thing to note is that 3D Geometry is the only element to receive a "minimum" value of 6 . The mean rating for $3 \mathrm{D}$ Geometry was 6.94 , which is the highest of any of the elements.

\begin{tabular}{|c|c|c|c|c|c|c|c|}
\hline$\#$ & Field & Minimum & Maximum & Mean & $\begin{array}{l}\text { Std } \\
\text { Deviation }\end{array}$ & Variance & Count \\
\hline 1 & 1 & 6.00 & 7.00 & 6.94 & 0.24 & 0.06 & 51 \\
\hline \multicolumn{8}{|c|}{ Q7 - Dimensional Information } \\
\hline$\#$ & Field & Minimum & Maximum & Mean & $\begin{array}{l}\text { Std } \\
\text { Deviation }\end{array}$ & Variance & Count \\
\hline 1 & 1 & 1.00 & 7.00 & 5.04 & 1.94 & 3.76 & 51 \\
\hline \multicolumn{8}{|c|}{ Q8 - Detailed Product Geometry } \\
\hline$\#$ & Field & Minimum & Maximum & Mean & $\begin{array}{l}\text { Std } \\
\text { Deviation }\end{array}$ & Variance & Count \\
\hline 1 & 1 & 1.00 & 7.00 & 6.20 & 1.44 & 2.08 & 50 \\
\hline \multicolumn{8}{|c|}{ Q9 - Revision/Version History } \\
\hline$\#$ & Field & Minimum & Maximum & Mean & $\begin{array}{l}\text { Std } \\
\text { Deviation }\end{array}$ & Variance & Count \\
\hline 1 & 1 & 1.00 & 7.00 & 5.18 & 1.95 & 3.79 & 51 \\
\hline
\end{tabular}

Figure 13: Questions 6-9 on Element Rating (N: 49-50) 
Q10 - Geometric Dimensions and Tolerances (GD\&T)

\begin{tabular}{|lccccllll} 
\# & Field & Minimum & Maximum & Mean & $\begin{array}{l}\text { Std } \\
\text { Deviation }\end{array}$ & Variance & Count \\
\hline 1 & 1 & 3.00 & 7.00 & 6.08 & 1.27 & 1.60 & 51 \\
\hline $\begin{array}{l}\text { Q11 } \\
\text { \# }\end{array}$ & Field & Minimum & Maximum & Mean & $\begin{array}{l}\text { Std } \\
\text { Deviation }\end{array}$ & Variance & Count \\
\hline 1 & 1 & 2.00 & 7.00 & 5.37 & 1.45 & 2.12 & 51 \\
\hline
\end{tabular}

Q12 - Surface Finish/Characteristics

\begin{tabular}{|llllllll|}
\hline \# & Field & Minimum & Maximum & Mean & $\begin{array}{l}\text { Std } \\
\text { Deviation }\end{array}$ & Variance & Count \\
\hline 1 & 1 & 1.00 & 7.00 & 5.22 & 1.64 & 2.68 & 51 \\
\hline
\end{tabular}

Q13 - Engineering Notes

\begin{tabular}{|llllllll|}
\hline \# & Field & Minimum & Maximum & Mean & $\begin{array}{l}\text { Std } \\
\text { Deviation }\end{array}$ & Variance & Count \\
\hline 1 & 1 & 2.00 & 7.00 & 5.25 & 1.54 & 2.39 & 51 \\
\hline
\end{tabular}

Figure 14: Questions 10-13 on Element Rating (N: 51)

Q14 - Bill of Materials

\begin{tabular}{|llllllll|}
\hline \# & Field & Minimum & Maximum & Mean & $\begin{array}{l}\text { Std } \\
\text { Deviation }\end{array}$ & Variance & Count \\
\hline 1 & 1 & 0.00 & 7.00 & 4.98 & 1.75 & 3.06 & 50 \\
\hline
\end{tabular}

Q15 - Referenced/Related Appropriate Standards

\begin{tabular}{|c|c|c|c|c|c|c|c|}
\hline$\#$ & Field & Minimum & Maximum & Mean & $\begin{array}{l}\text { Std } \\
\text { Deviation }\end{array}$ & Variance & Count \\
\hline 1 & 1 & 1.00 & 7.00 & 4.71 & 1.87 & 3.51 & 49 \\
\hline \multicolumn{8}{|c|}{ Q16 - Change Management Data } \\
\hline$\#$ & Field & Minimum & Maximum & Mean & $\begin{array}{l}\text { Std } \\
\text { Deviation }\end{array}$ & Variance & Count \\
\hline 1 & 1 & 1.00 & 7.00 & 4.35 & 1.95 & 3.82 & 49 \\
\hline \multicolumn{8}{|c|}{ Q17 - Product Specifications } \\
\hline$\#$ & Field & Minimum & Maximum & Mean & $\begin{array}{l}\text { Std } \\
\text { Deviation }\end{array}$ & Variance & Count \\
\hline 1 & 1 & 1.00 & 7.00 & 4.34 & 1.86 & 3.46 & 50 \\
\hline
\end{tabular}

Figure 15: Questions 13-17 on Element Rating (N: 49-50)

Another important finding is that Product Specifications had the lowest mean of all 12 elements, showing that even though this element is important, it is not important for everyone to have the product specifications. The IDEF0 diagrams created as a part of Stage Three show where some of these information elements fit into the workflows; however, not every information element was utilized directly as a part of the participants' workflows. 
Table 3: Question 19 Data

\begin{tabular}{|c|c|c|c|c|}
\hline Question & In Model & In Drawing & Both & Total \\
\hline 3D Geometry & 36 & 0 & 15 & 51 \\
\hline $\begin{array}{c}\text { Dimensional Info. } \\
\text { Detailed Product } \\
\text { Geometry }\end{array}$ & 25 & 9 & 17 & 51 \\
\hline $\begin{array}{c}\text { Revision/Version History } \\
\text { Geometric Dimensions } \\
\text { and Tolerances }\end{array}$ & 17 & 2 & 16 & 51 \\
\hline $\begin{array}{c}\text { Materials } \\
\text { Specifications/Definitions }\end{array}$ & 18 & 12 & 22 & 50 \\
\hline $\begin{array}{c}\text { Surface } \\
\text { Finish/Characteristics }\end{array}$ & 22 & 4 & 25 & 51 \\
\hline $\begin{array}{c}\text { Engineering Notes } \\
\text { Bill of Materials }\end{array}$ & 14 & 9 & 20 & 51 \\
\hline $\begin{array}{c}\text { References/Related } \\
\text { Appropriate Standards }\end{array}$ & 13 & 15 & 22 & 51 \\
\hline $\begin{array}{c}\text { Change Management } \\
\text { Data }\end{array}$ & 16 & 20 & 18 & 51 \\
\hline Product Specifications & 14 & 14 & 21 & 51 \\
\hline
\end{tabular}

Question 19 in the Delphi survey Round 3 asked participants to choose one option out of three for each of the twelve information elements listed. Participants could select that the element needs to be present in the model, in the drawing, or both as seen in Table 3. Question 19 was designed to see whether the information elements that emerged from the previous rounds of the surveys and the interviews were as important for inclusion in a model as they were for inclusion historically in a drawing. The only elements deemed more important to the drawing (and not the model) were "Engineering Notes, Bill of Materials, References/Related Appropriate Standards and Product Specifications". These specific information elements are typically defined as text, and textual notes have been historically difficult to display within a 3D model environment due to the default pictorial orientation of a model and the human difficulty in reading text and other symbols that are not parallel to the computer screen. In a paper-based drawing, the orientation of textual information was always parallel to the presentation medium (i.e., the plane of the paper on which the drawing was made). Thus, a human was always able to read the information in a note, bill of materials, or specifications since the visual presentation of the information was congruent with the function of the human visual system.

Many of the information elements addressed in Question 19 were identified as important in both the model and the drawing, but the element that was MOST important to both definitions was "Materials Specifications/Definitions". Question 19 helped answer an underlying theme of our study: which information elements are important to which definition (model or drawing)? Most of responses indicate that the 3D Geometry is in fact the most important element, across all three rounds of the Delphi and between models and drawings. 
The final four questions in Delphi survey Round 3, shown in Table 4, asked about the ability to adopt MBD/MBE based on culture, education, infrastructure and technology. In all four questions, the mean of the responses (51 in total for each question, 50 for question 25) came back to above 4.5. On a seven-point scale, this puts all the responses into the category that these factors do influence the ability to adopt MBD/MBE. These questions served as guidance for the next round of interviews.

Table 4: Questions 22-25 of Delphi Round Three

\begin{tabular}{|c|c|c|c|c|c|c|}
\hline Question & Minimum & Maximum & Mean & $\begin{array}{c}\text { Std. } \\
\text { Deviation }\end{array}$ & Variance & Count \\
\hline $\begin{array}{l}\text { Q22 - To what extent } \\
\text { is the lack of } \\
\text { education a factor in } \\
\text { the ability of a } \\
\text { company to adopt } \\
\text { MBD/MBE? }\end{array}$ & 1 & 7 & 4.65 & 1.80 & 3.25 & 51 \\
\hline $\begin{array}{l}\text { Q23 - To what extent } \\
\text { does corporate } \\
\text { culture factor into } \\
\text { the ability of a } \\
\text { company to adopt } \\
\text { MBD/MBE? }\end{array}$ & 2 & 7 & 5.92 & 1.31 & 1.72 & 51 \\
\hline $\begin{array}{l}\text { Q24 - To what extent } \\
\text { does the availability } \\
\text { of proper technology } \\
\text { infrastructure affect } \\
\text { the adoption of } \\
\text { MBD/MBE? }\end{array}$ & 2 & 7 & 5.41 & 1.50 & 2.24 & 51 \\
\hline $\begin{array}{l}\text { Q25 - To what extent } \\
\text { does having a } \\
\text { relevant data } \\
\text { architecture affect } \\
\text { the adoption of } \\
\text { MBD/MBE? }\end{array}$ & 2 & 7 & 5.00 & 1.33 & 1.76 & 50 \\
\hline
\end{tabular}

\subsection{Delphi survey Round Three Interview Data}

In total, six interviews took place for Delphi survey Round 3 and to contextualize the IDEF0 diagrams developed on Stage Three. The interviews were a hybrid style, starting with the interview questions for a structured and guided response path, ending with the IDEF0 diagrams and a lot of criticism and feedback about the structure of the research study and the survey results to date. The first part of the interviews contained information relevant to Delphi survey Round 3, and asked for feedback based on the results discussed in Delphi survey Rounds 1 and 2. The first, and most prevalent, response from the interviewees at this stage was the confirmation that 3D Geometry, in any form, is the most important information element, 
regardless of the presence of any supporting information. However, this response was typically followed with a discussion about where most companies are in their adoption process for 3D tools. Original equipment manufacturers and suppliers are currently at a stage where 2D Geometry is still extremely relevant, and is perceived to be necessary in their internal processes. The consumers of digital product data in their respective supply chains may not have the ability to use CAD software or have capabilities to read a 3D model, so for some of these participants 2D Geometry and sketches are very important. The use of a CAD system requires a license for that system, which can be pricey. This means that smaller businesses, suppliers, or OEMs may not be able to afford a CAD license to read the data sent to them by a customer or partner. In this way, being able to transmit all necessary information in a $2 \mathrm{D}$ format and without the use of a CAD system, they can save money and still perform their business.

The interviewees described all twelve elements identified and expanded upon in Delphi survey Round 2 as imperative. Although most of the participants only interacted with one or two of the information elements actively inside of their workflows, they knew that the other information elements were imperative to the job roles before and after their position. Even though the information elements were all present at the interviewee's position in the product lifecycle, they were imperative at different points in the lifecycle. This finding is congruent with the minimum information model, as the information utilized by each workflow was slowly emerging as a combination of domain knowledge and the common information elements. And the model-based definition concept is becoming a more common structure of information dissemination.

Another emerging theme from the Delphi surveys and interviews is adopting modelbased definition has a substantial cost - not just in money, but also in time, which is one of the largest concerns in industry, especially when a large, distributed organization is trying to implement MBD tools and methods. An adequate computing infrastructure is also important, as is having the correct software that suits the needs of the company. Having the ability to install and set up this infrastructure and software, as well as properly training employees to use MBD tools and methods, is a formidable task. Training employees to utilize MBD methods and tools correctly, and to genuinely revolutionize their company though an MBE transformation, is particularly stressful, as training typically happens in tiers. In the Delphi survey Round 3 interviews, some training was described as a series of gates or milestones. As each "gate" opens and training is brought down another level, there is room for error. There is also room for each level of training to be changed slightly, based on one person's preferences over another. Human error is a substantial barrier to adopting MBD, as training for use and implementation of MBD tools and methods can take much more time and effort in the data authoring stages of the lifecycle versus the data consumption stages.

A common implementation and training problem is that MBD deliverables are not always mature enough, and not everyone in the company has access to a workstation with CAD software available. This means that even if they are trained to understand and utilize the software, or the information presented in the 3D model, they may not have access to it in the same form in which they were trained to use it. Deploying computing architecture to job roles where it has 
historically not been needed can be a difficult discussion to have for many manufacturing or quality managers. One medium of information transfer that is becoming more popular is the use of such files as 3D PDF, STEP, or JT [23-26], allowing anyone with a proper viewer to open, read and interrogate 3D Geometry information and other MBD information associated with that model. This makes the information formerly limited by software more accessible to everyone in the process, and relieves some of the tension brought on by budget and training previously. Again, the communicative power and time savings for using MBD tools and methods are in the data consumption stage and not the authoring stage.

The final discussion held in the interviews was about the lack of understanding surrounding the concepts of model-based definition and model-based enterprise, as well as the methods and tools that accompany each one. These tools and methods are used to increase data flow and data integrity throughout an organization. They can be used to narrow down processes and eliminate errors caused by multiple master definitions, and of course they can help identify what information is needed, and when it is needed, to reduce the impact of data overload on each employee. The discussion that very few companies are having currently is whether they need to move towards creating an environment to use MBD tools and methods, or if it is even practical for them to do so. While MBD and MBE sound great in concept, they are not always the solution a company needs in the immediate sense. In fact, some companies or organizations do not have the resources or knowledge necessary to move from their current system to an all-digital product data architecture at once. They need to be able to move in stages with a demonstrable ROI indicator. Understanding if using MBD and MBE tools and methods is right for the company should be the first step. Implementation, training and infrastructure should only be considered after it has been decided that MBD methods are the path forward.

\section{Stage Three: Using IDEF0 Models to Contextualize the Minimum Information Model}

After identifying the relevant and significant information items by way of the Stage 1 and Stage 2 surveys and interviews, it was necessary to capture the relationships between these elements in an information framework model. The Integrated Definition (IDEF) model was used to present the minimum information model elements in each workflow. The IDEF model's framework has 16 level of detail in which processes and information can be documented. This study only used the IDEF0 stage of the framework, which are used to model the functions of an enterprise. A graphical model, like a flowchart, is created which illustrates the major activities in a process. IN this research study, the major activities in each workflow represent the stages in that process in which information was exchanged. The IDEF diagramming process has a formal structure, showing arrows and labels in specific places with specific meaning: what controls the function, who performs it, what resources are used in carrying it out, what it produces, and what relationships it shares with other functions.

In Figure 16, it shows an example of how IDEF0 diagrams work. The left side arrows describe inputs, the top arrows describe controls (or things that limit the process and task 
activity), the bottom arrows are mechanisms (things utilized to complete the process and task activity) and the right-side arrows are outputs of that process and task activity. The IDEF structure was explained to all the participants before the discussion about the IDEF0 diagrams began, and all questions were answered. The IDEF0 diagrams were created to visualize the workflows identified in Stage One and to help understand where the minimum information model elements and the common information elements fit into these workflows. The interviews provided a sense of external validity that the original version of each IDEF0 was accurate, and that the revised version reflected the feedback provided as well as the implementation of MIM elements. It was emphasized to the interview participants and that these diagrams, while carefully constructed and revised, are an initial presentation at visualizing these workflows and may not be completely accurate in their current form according to industrial practice. All IDEF0 diagrams created from this research are subject to changes as more data are gathered in the future, and as the workflows are further scrutinized.

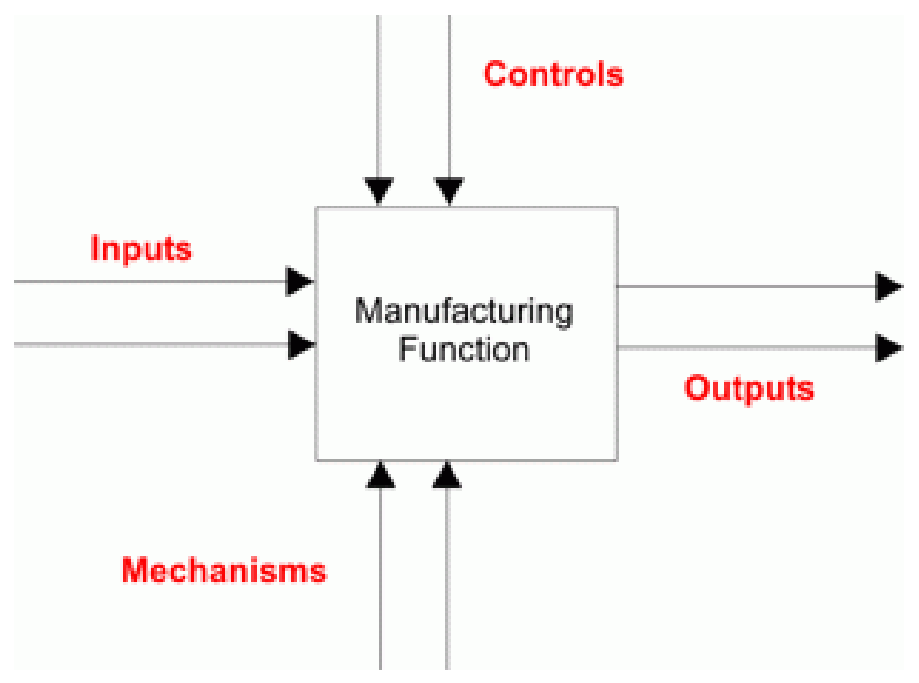

Figure 16: IDEF0 Representation

Although the workflows identified in Stage One of this study are four separate processes, they typically follow each other sequentially in a product's lifecycle, and are often connected by sharing a first or last step or by way of the information artifacts they share. The diagrams shown in Figure 17 through Figure 24 were created in Microsoft PowerPoints as a preliminary draft of what they should be. Innoslate, an online web-based toolset that allows for the creation and manipulation of IDEFx visualizations was used later to reconstruct these IDEF0 diagrams for archival purposes and to enable more accurate editing of them in the future.

One response shared by all participants unanimously was that having a map of information to follow allows for the tracking of information throughout the entire workflow or set of workflows. If each workflow were broken down beyond what is presented, and an in-depth look was taken to understand a company's process for information dissemination, then the understanding of data flow inside of each workflow would be far greater than it has ever been. In their current state, each IDEF0 diagram has five main "activities" inside of them, with inputs, 
controls, outputs and mechanisms unique to each activity. These activities could be further decomposed to include every piece of information included and therefore refine the processes shown based on the company that is using them. However, in their current state, the IDEF0 diagrams are created to give an overview of each workflow with MIM and CIM information inserted and shown. In-depth depictions each process could happen as part of a later research project, but they were outside the scope of this current work.

Integrated Product Teams were brought up in the interviews and describes the bringing of the design and manufacturing teams to the front of the creation of a product. Rather than having separate phases (ideation, design, prototype, manufacture), all the phases are tied together and the teams collaborate from the start. This prevents errors during the design and ideation phases, and helps the manufacturing team prepare for the product that is to be created.

Machinists, technicians and engineers all work together to build the product designed and created early, so having them involved from the start is imperative and was a change made in the IDEF0 diagrams. This early implementation meant that many of the MIM and CIM terms would appear sooner in the workflows rather than later, as they are being considered up front rather than at their own individual slots inside of each workflow.

For example, in Figure 17 and Figure 18, 3D Geometry is an element that is created early and modified often, regardless of whether it is the master digital product definition or not. Typically, the 3D model would be created after the conclusion of the Concept to Prototype workflow, and is an output of its final stage. However, the participants that reviewed the IDEF0 diagram for that workflow pointed out that the model is generated much earlier in the workflow, often becoming an output of the second stage of that workflow, allowing the model to be modified and changed earlier in the process. In doing so, the model remained in a refined and solid state by the time it reaches the start of the Prototype to Detailed Product Definition workflow. 


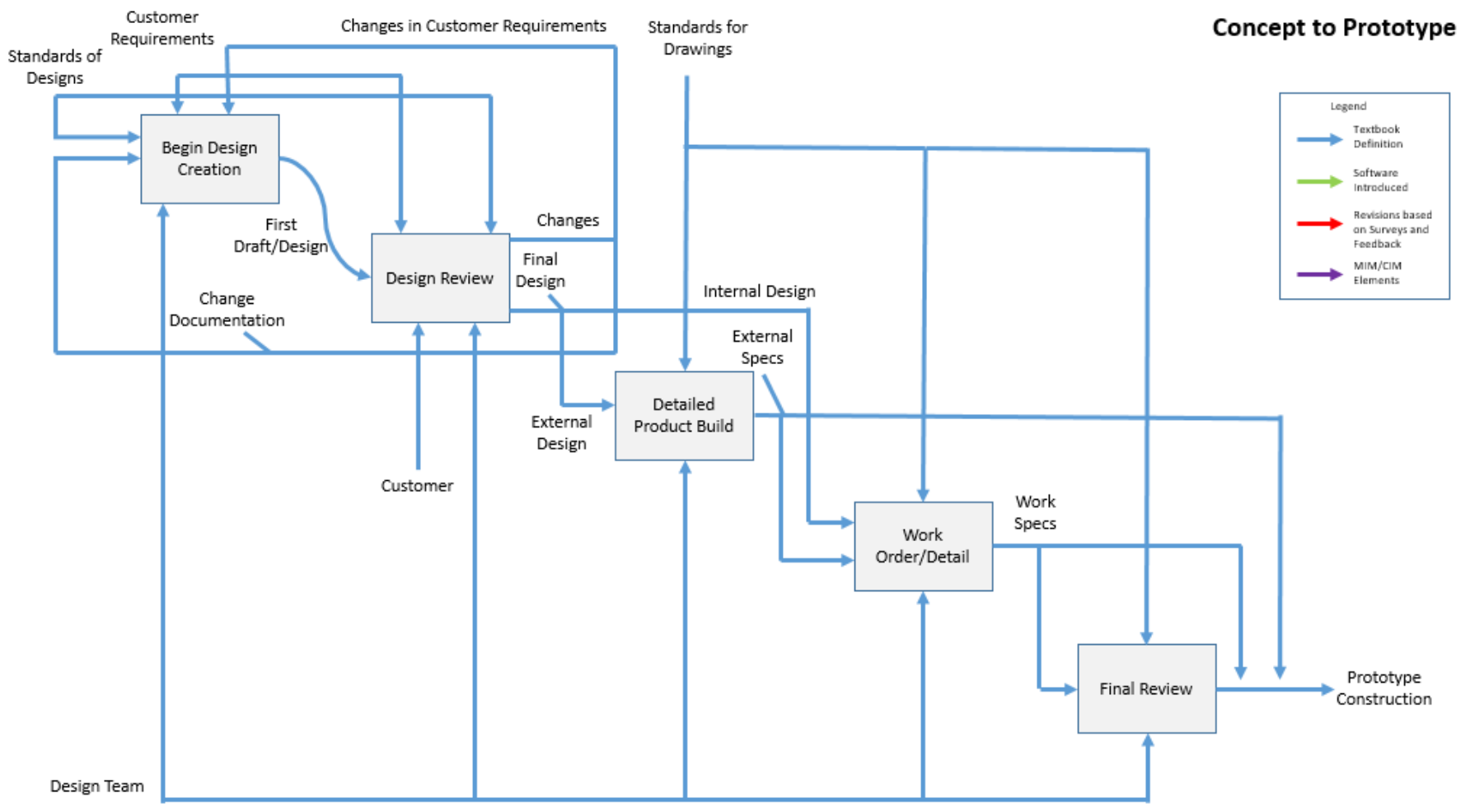

Figure 17: Concept to Prototype IDEF0

Figure 17 shows the first IDEF0 diagram (Concept to Prototype) created to represent the flow of information inside of the workflow. Like Figure 19 and Figure 20, this diagram underwent scrutiny to verify its integrity. The key changes that were resolved from the interview feedback are represented in Figure 18. Two diagrams exist for each workflow, the first being the "textbook definition" of the model, while the second reflects changes made by people in industry providing feedback against the textbook definition. The resulting diagram is fuller, complete, and tailored towards the goal of understanding where the MIM and CIM information fit into the diagrams. Each person interviewed shared insight into how these workflows work specifically inside of their industry or company, the result was that every industry and company has their own way of getting their products finished. There is no static "process" that is followed in terms of data flow, it is customized to fit the culture and needs of the company. 


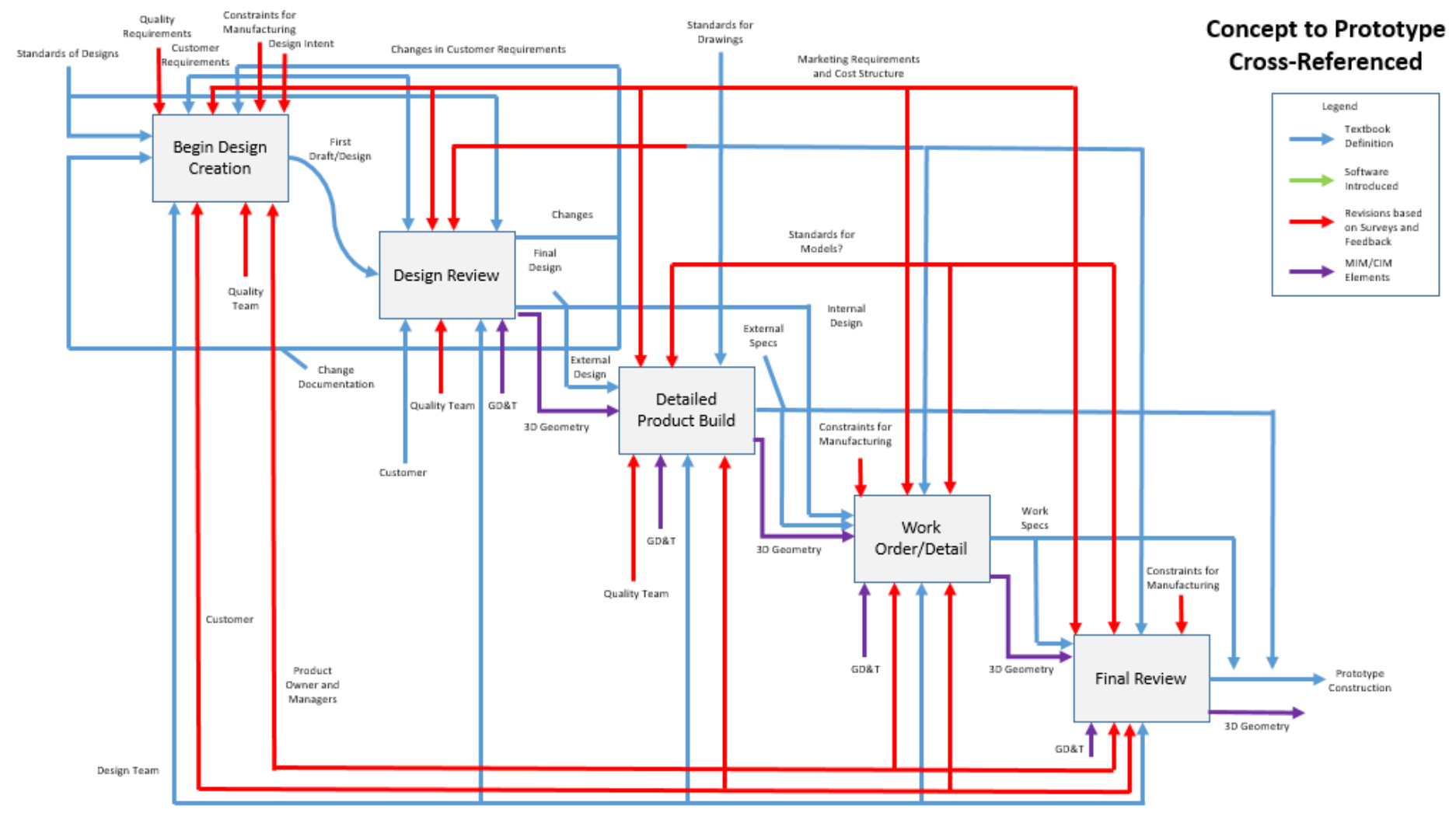

Figure 18: Concept to Prototype IDEF0 Revised

This Concept to Prototype workflow shows the creation of the work order that goes into the creation of the prototype. A key lesson taken from the interviews was that earlier on in the process the quality, manufacturing and customer requirements are considered, the smoother the output will be for the product. Notice how, in the Concept to Prototype workflow in Figure 18, the mechanisms introduced are the customer, quality, managers, and as time goes on, the GD\&T of the product that is created digitally. The middle three activities begin the review process for the design that was output from the design creation phase. These three activities are crucial for reforming the specs necessary on the product as well as the eventual work order that gets produced to create the prototype. Figure 19 and Figure 20 detail the creation of the detailed product definition. 


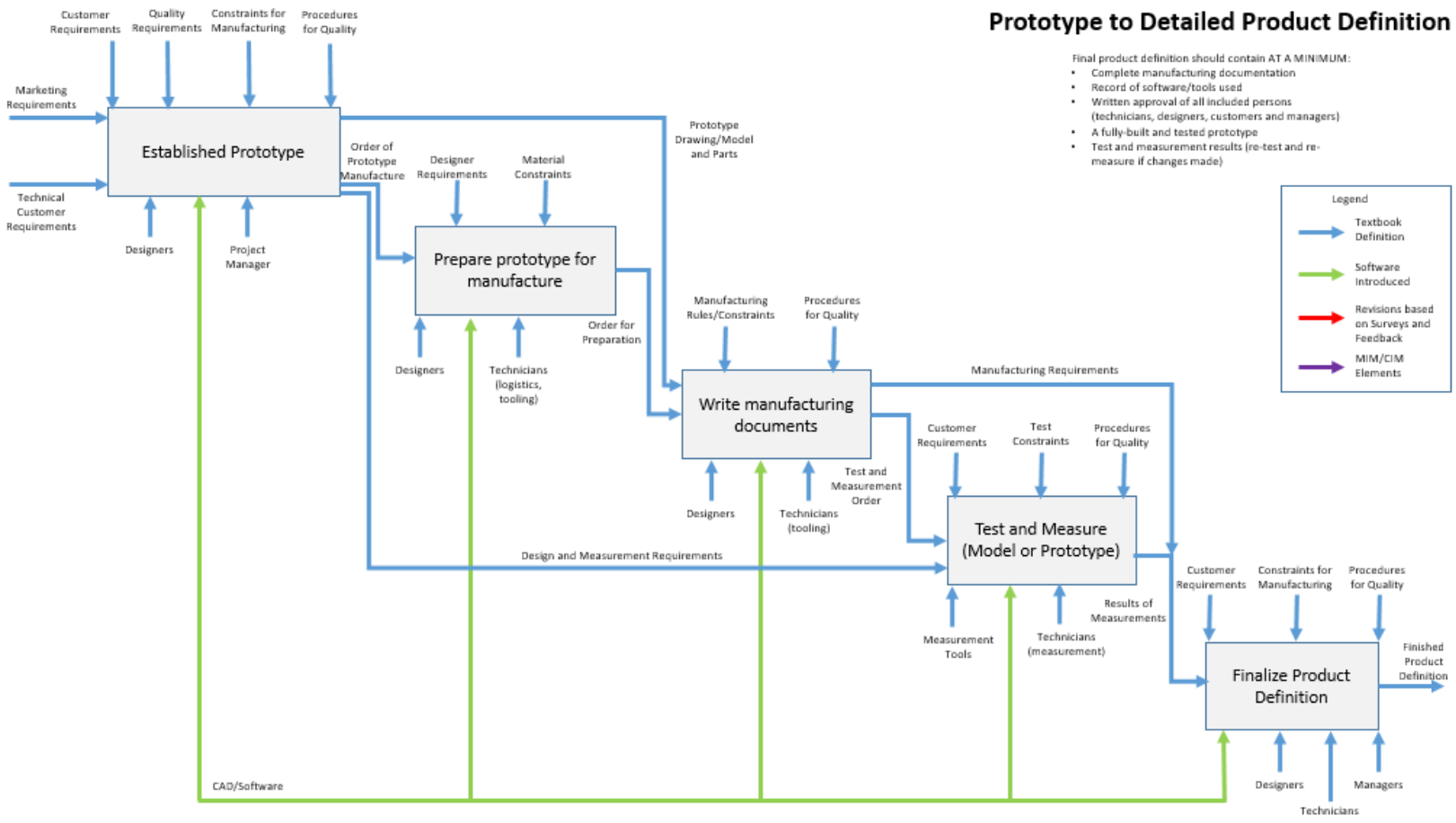

Figure 19: Prototype to Detailed Product Definition

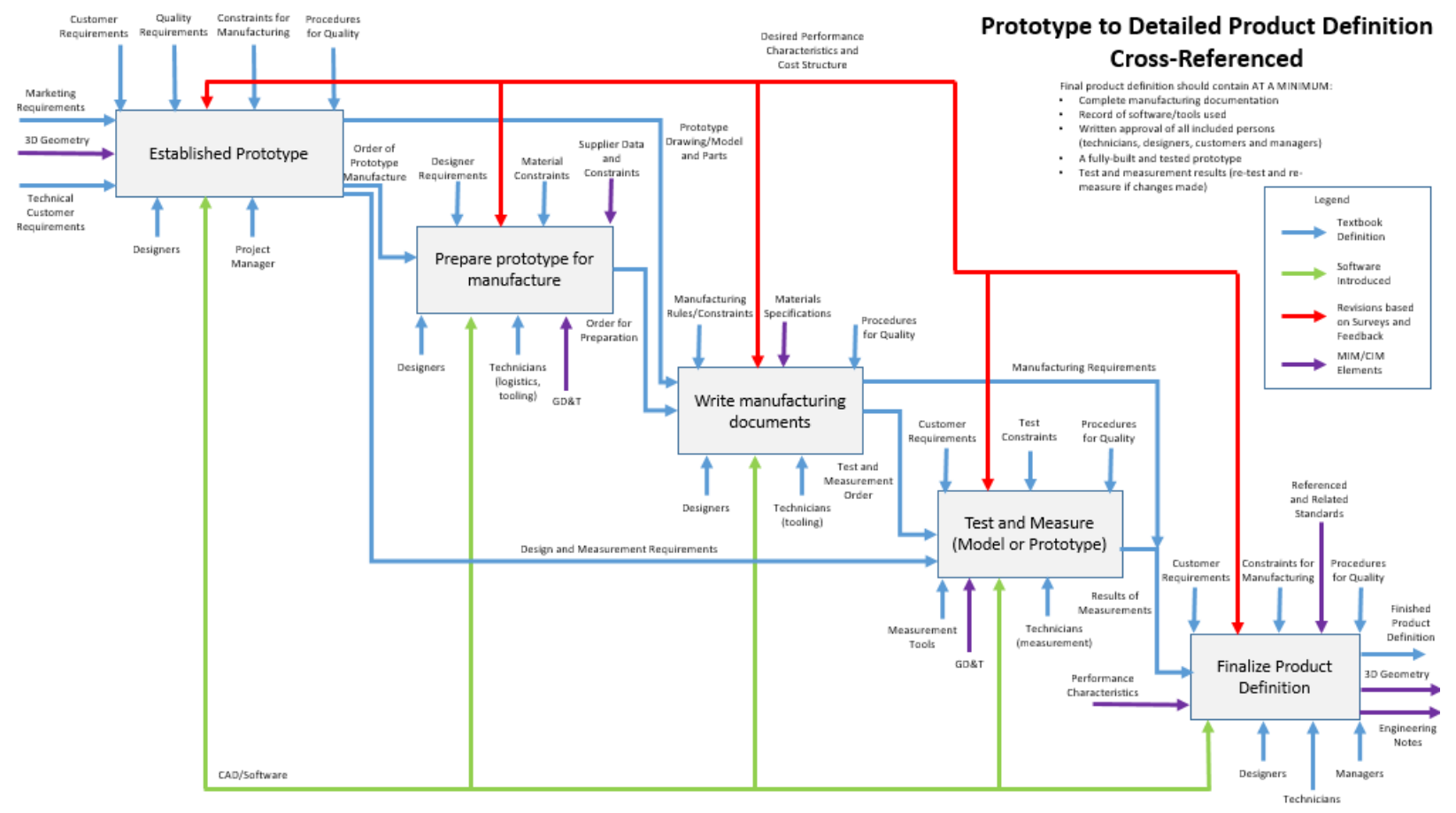

Figure 20: Prototype to Detailed Product Definition Revised

Figure 20 is the revised version of the second set of IDEF0 diagrams created. This diagram in specific introduces the software and constraints of different departments on the data 
that flows through. Preparing a prototype is a process that used to take a lot of time and resources, however, it has been seen that 3D models typically replace the need to build a prototype. Thanks to processes like finite element analysis (FEA) the real-world conditions that products would undergo can be simulated. The 3D geometry flows through the Prototype to Detailed Product Definition diagram rather than being the main component, as some companies have chosen not to, or haven't had the resources to move to a model-based enterprise. The main output of the Prototype to Detailed Product Definition workflow is a finished product definition, meaning it is understood what this product is going to be and how it should be defined. The main change to observe for the Prototype to Detailed Product Definition workflow diagram from the textbook was the addition of the testing analytics for the prototype that enter the discussion and consideration at the "established prototype" function in the top left of Figure 20.

Starting from the first activity of the workflow shown in Figure 20, designers and project managers are the main mechanisms while the constraints are those imposed from the customer, quality and manufacturing. Activities two and three, preparing the prototype for manufacture and the writing of the manufacturing documents, have the project manager dropped from the mechanisms and have instead introduced technicians. One key point of feedback from the interviews suggested that specialists will be consulted during these activities, specialists in the tools, the process, the logistics, etc. The constraints imposed on activity two introduce the requirements of the designer and materials, while on activity three the manufacturing rules and quality procedures are the main constraining factors. CAD software and other software are introduced in all five activities of this workflow as needed, serving as mechanisms to complete the product definition. An order for testing and measuring is the output of activity three, as activity four goes into the testing of the prototype for quality and endurance. One important addition here is that manufacturing requirements are an output directly of activity three, meaning that when the measurements are taken and the results are gathered, the limitations of manufacturing will be considered. The final activity of this workflow produces a finished product definition and gets sent off for manufacturing. In the revised version of the workflow (Figure 20), cost structure and desired performance are included as constraints due to budgetary requirements and meeting expectations for product use. The final activity reintroduces managers, as approvals are needed as the final barrier to manufacture. This diagram flows directly into Figure 21 and Figure 22. 


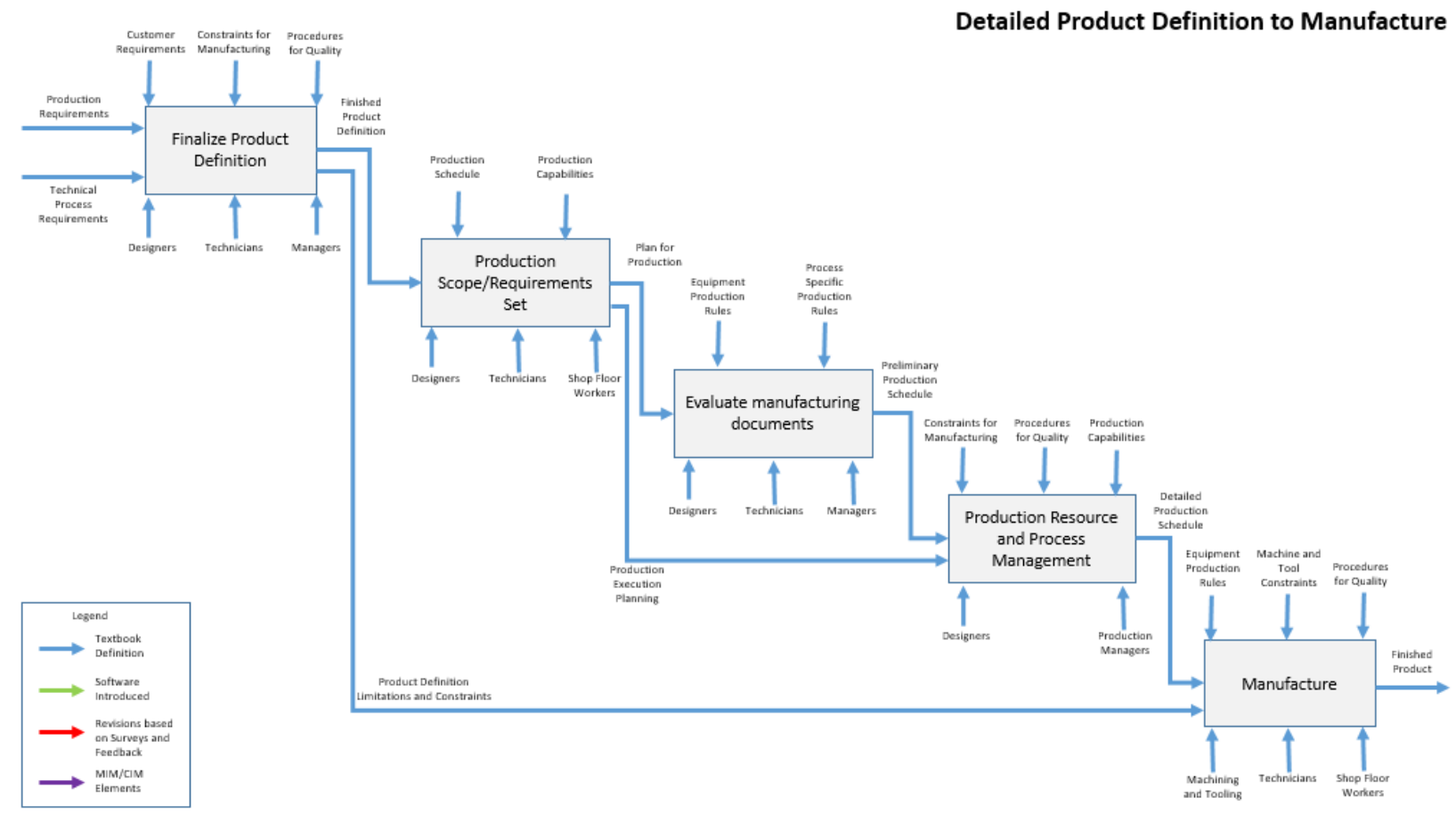

Figure 21: Detailed Product Definition to Manufacture

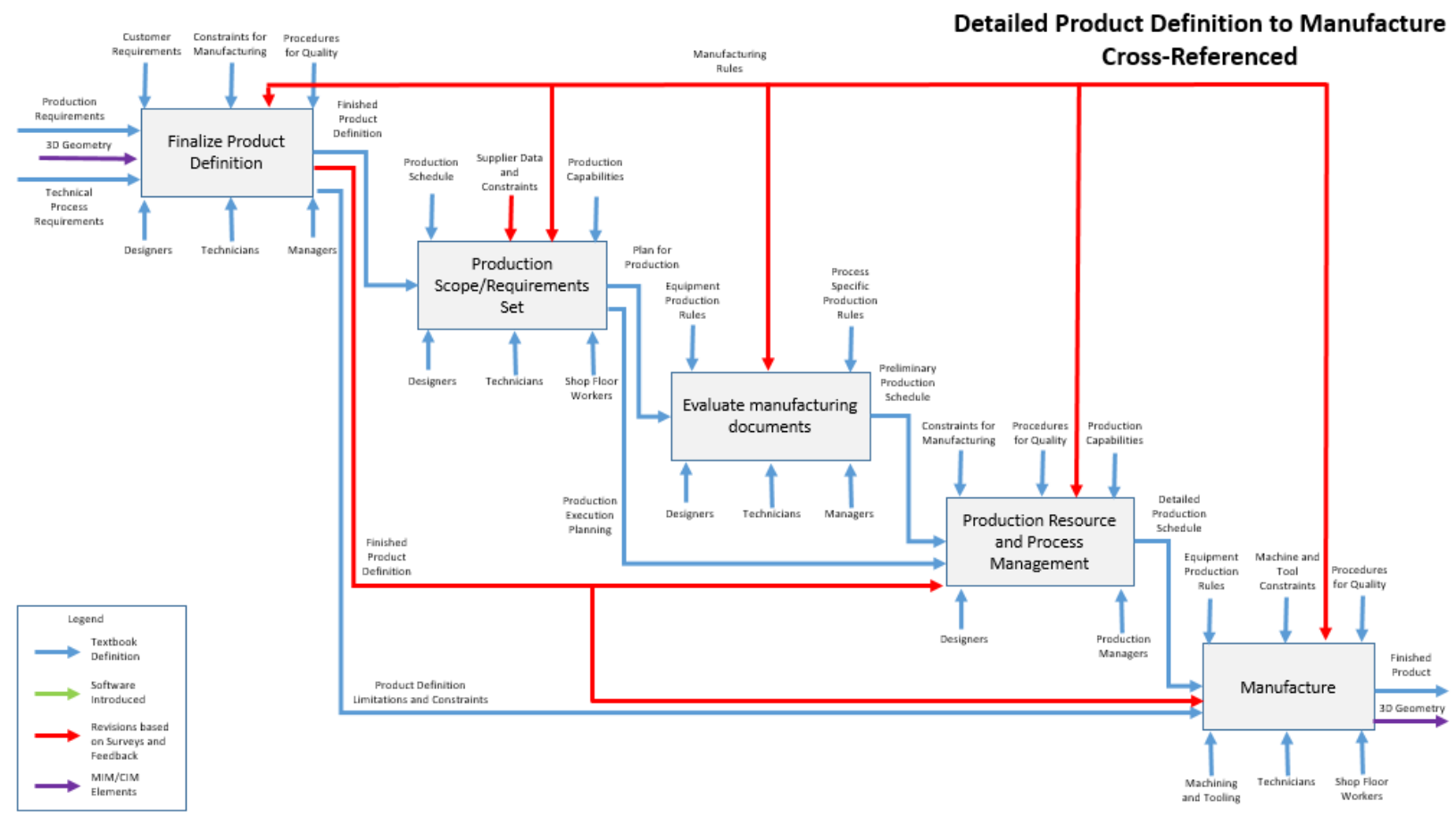

Figure 22: Detailed Product Definition to Manufacture Revised

In Figure 22, the product definition flows directly into the first activity of this diagram and is immediately constrained by the customer, manufacturing rules and quality procedures. 
These constraints keep the product definition in check, and the revisions suggested by the study's results and the interviews introduced the flow of the finished product definition to other major parts of the workflow. The product definition, while becoming a main component of the production scope/requirements, also becomes part of the production resource and process management tabs, as well as the manufacture tab.

Take note of the mechanisms introduced at each phase of this workflow. At first, designers, technician and shop floor workers are the main involved parties. However, at the third activity, managers take over as a key mechanism, replacing shop floor workers. It is assumed that at this point in the workflow, the documents defining production are being created and finalized, which often requires managerial input on multiple fronts. The fourth activity drops technicians and replaces managers with production managers. The production managers know the limits of production and in the end, are the ones responsible for managing the production of the product, so their input is critical here. The final activity, manufacture, reintroduces shop floor workers but also the machining and tooling components that will be used to create the product. The finalized product is the end-result of this workflow and flows directly into Figure 23 and Figure 24.

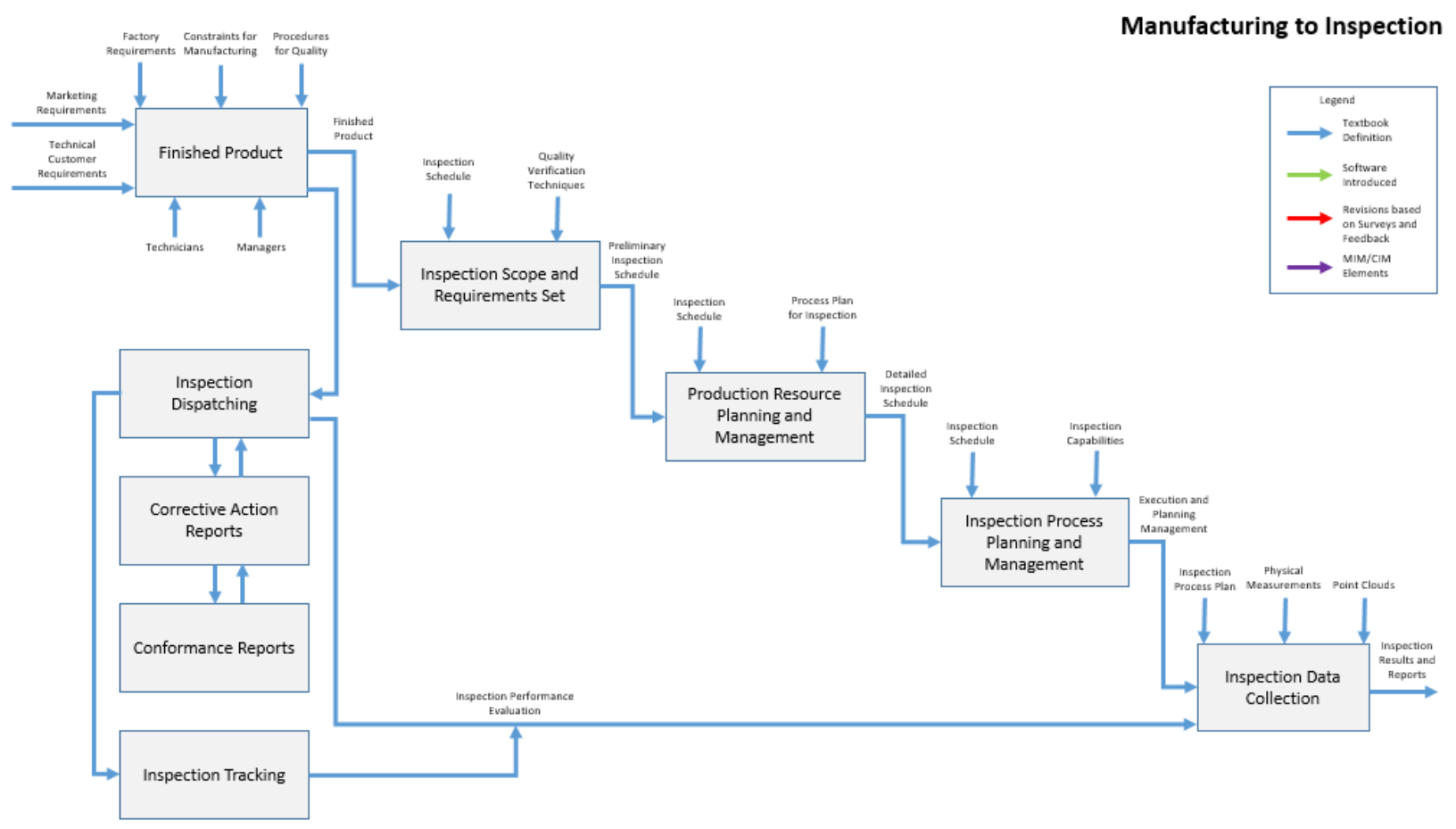

Figure 23: Manufacturing to Inspection IDEF0 


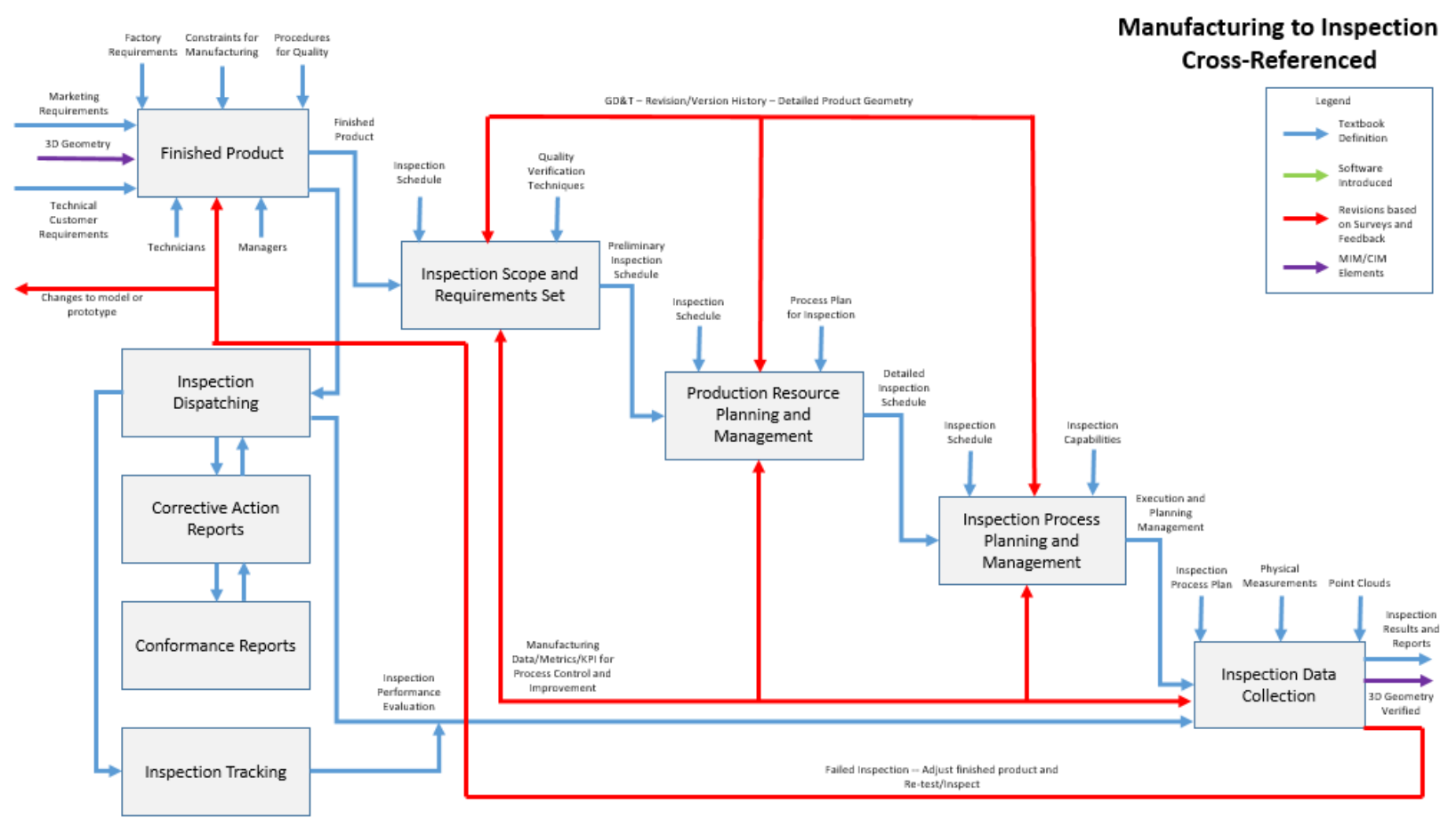

Figure 24: Manufacturing to Inspection IDEF0 Revised

The finished product is the first activity of the Manufacturing to Inspection workflow, serving as the base for the inspection workflow that is beginning. The original workflow created in Figure 23 shows how a sub-workflow is created that reflects on the history of the product, where all faults and changes are stored. A verification of conformance reports is implemented to reflect whether the problems associated with the product were fixed and documented correctly. If it passes this phase, it moves on to the inspection tracking phase, being put on hold until the Manufacturing to Inspection workflow has finished. The second activity in the Manufacturing to Inspection workflow introduces manufacturing data/metrics/kpi for process control and improvement as a mechanism. The new constraints introduced are the GD\&T along with the revision/version history in the detailed product geometry. Activities three and four of the Manufacturing to Inspection workflow begin the processes for inspection, gathering production documentation and the capabilities of the inspection based on available equipment and personnel. Once everything is prepared, the inspection process is implemented, being constrained by things such as physical measurements and the inspection process plan that was created. Pending a successful inspection, the results and reports are generated along with the finalized 3D Geometry. If the inspection is failed, it is sent back to the beginning of the inspection cycle for a re-test and then any changes that need to be immediately made to the model or prototype are considered.

This workflow serves as the "catch all” for mistakes made earlier in the process. This is because during the inspection phase, a critical analysis is run of the product's history, checking to make sure all fixes specified were completed and that all approvals were gathered and documented. It can be assumed that most companies want to avoid revisions and changes to the processes at this point, as those changes can be costly. The interviews introduced some key 
changes to all workflows in question that improve the integrity of the product from the start. If errors are made, they are often caught in this final workflow. If a faulty product makes its way to market, recalls are then performed when defects are discovered (if necessary). All failed inspections undergo a lengthy re-test and re-evaluation of the product at early phases.

Stage one and Stage two of this study worked together to gather information about industry, specifically the four workflows defined, to generate a better understanding of how information is utilized inside of industry. Time is money, so goes the old saying. If workflows are bogged down by unnecessary steps, data, or otherwise unnecessary people or departments then time is being wasted. Stage one and Stage two identified four workflows and how the users inside of those workflows utilize the data generated, or perhaps generate the data used. One key factor of the IDEF0 diagrams created was the distinction between "textbook" and revised workflows. The revised workflows were heavily influenced not just by interviews, but also by the findings from the first two stages. For example, stage one discussed how implicit information Is different from explicit information and why that matters in terms of readability and interpretability. Stage two sought out specific elements and their uses, what form those elements needed to be present in to be most effective and how those elements were consumed. Combined, those two concepts work together to help understand where the elements in stage two are generated from and whether they should be defined explicitly or implicitly. The IDEF0 diagrams then represent the elements as best as possible in terms of where they are inserted and how they flow. Many changes were made between the pairs of IDEF0 diagrams, but all changes were influenced by a combination of stage one, stage two, stage three and the interviews.

One of the final comments from the interviews was about constraints and where they should be implemented. In earlier versions of the workflows, constraints (controls in the context of IDEF diagrams) were imposed at later stages or at the very beginning when design was critical. It was mentioned that these constraints do not simply start and stop at specific stages, but typically influence the entire workflow from start to finish. Different constraints affect different stages, but they all work together to create a structured product that can be manufactured according to the limitations of the company, and is a model that follows all rules specified by OEMs, suppliers and regulations. Again, as a reminder, these IDEF0 diagrams are in their first iteration and are subject to change in the future. This stage of the research created these visualizations to better understand where MIM/CIM elements are present and to track the flow of data inside of the four workflows targeted.

\section{Minimum Information Model}

The concept of the minimum information model (MIM) is a major finding from this study. The minimum information model provides a framework that links factual domain knowledge with organizational knowledge common for most actors within the enterprise to form what is necessary to replace drawings within a given information workflow. It helps with the distribution of information, the propagation of meaning within the organization, and clarity for those job roles dependent upon the model-based definition for information. When a company utilizes MBD tools and methods, it can also utilize the MIM that emerges as a result. The MIM concept essentially implies that any given workflow that requires the authoring and consuming 
of model-based information will have its own unique MBD made up of shape, behavioral, and contextual information. This minimum information model concept [6,27], displayed in Figure 25 , seeks to provide the information required to enable a given workflow inside of the lifecycle. Each user of the MIM would have separate information, depending on their position in the lifecycle. However, common information (a key component to the MIM) is shared across all workflows and the entire lifecycle. Domain specific information is relevant only to the workflow of the current author or consumer and is not passed between lifecycle stages or workflows.

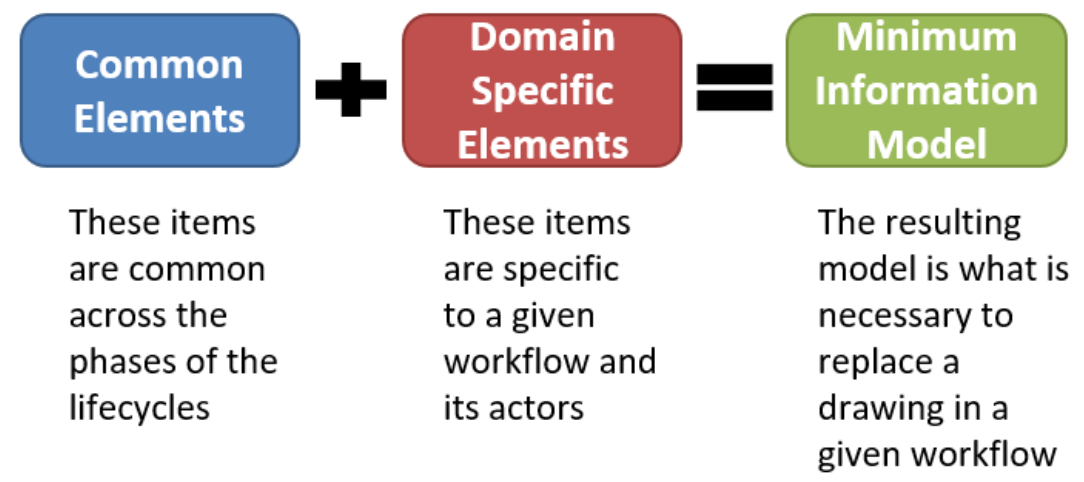

Figure 25: Minimum Information Model Diagram

Utilizing model-based definition and by extension, the MIM, is effective within manufacturing environments due to the information contained within it and the communicative power offered by the 3D model [28]. As some companies have successfully replaced 2D definitions with their $3 \mathrm{D}$ counterparts, and some are in the transition phase, understanding the required information elements necessary to enable that transition becomes important for effective and accurate communication. This is especially true for product-producing companies. It is also critically important for the software development community and the SDOs to identify and understand these information items as they develop tools to enable seamless data within the manufacturing environment.

Work has been done to analyze MBD and the effectiveness offered by digitalization. The results of this research found that improvements in the standardization of data and the visualization of processes needs improvement before the adoption of MBD as the master resource can be truly effective [29]. An initial step toward that is proper identification of the MIM and the information that it embodies. The overarching model that encompasses the MIM is the common information model (CIM). The common information model represents the data that is present throughout the lifecycle of a product, data that flows from start to finish. The data or elements present in the MIM may include common information, but will also include domain specific information that will not be passed to the next workflow or stage of the lifecycle. An example of this may be the section views or broken views of a 3D model that a designer uses to help verify the product definition. The designer will utilize standard views such as isometric, top, side, etc. but also may create their own views to help them understand the data they're working with or verify that their work was done correctly. No one outside of this specific task or workflow needs those extra information views, so they are not transferred to the next stage. 


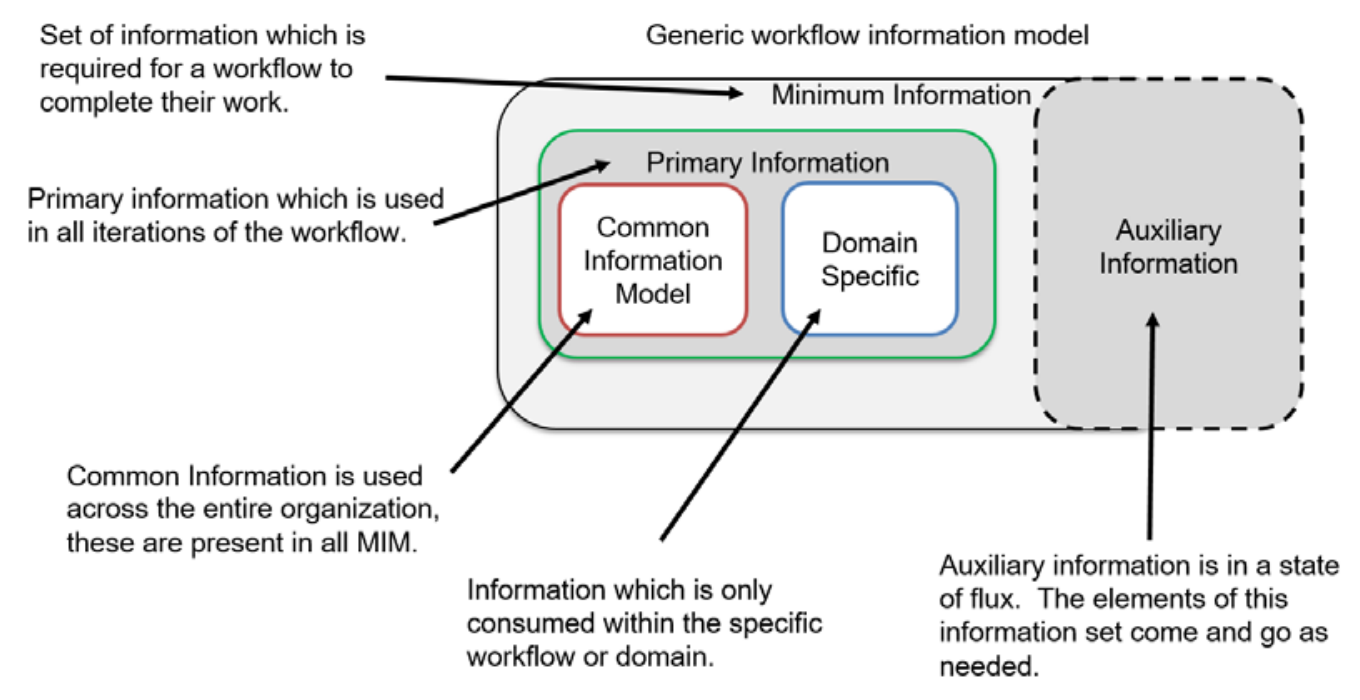

Figure 26: Primary and Auxiliary Information

This brings two different forms of information into the mix, primary and auxiliary, as seen in Figure 26. Primary information would be the standard views discussed before, especially the dimensions or tolerances associated with them. It is also the domain specific knowledge that is utilized inside of the workflow, knowledge that is common and utilized across multiple workflows. Auxiliary information is the extra information brought in to help the designer complete their workflow, in this case it would be the broken or section views created by the designer to help them understand the data and verify their work. Auxiliary information can vary widely from individual to individual, and usually does. Clear communication of the primary information is vital to the success of the MBD, as primary information touches a large portion of the population that will deal with the digital definition.

\section{Gaps and Opportunities}

This study sought to understand the way that information flows inside of workflows (specifically the four mentioned above), but it also strived to understand WHAT that information was and where it inserts itself into the process. A first major gap in the study is the spread of participation. Aerospace dominated the ranks in terms of response, but this isn't to say that Automotive, Defense/Military or any of the other industries have less of a voice in the overall discussion. This gap presents the opportunity to focus in on specific industries and after some data has come back, compare those results to other industries to see how the data holds up.

One important opportunity created by this work is in the realm of qualitative data. Another study should be conducted to understand where the twelve elements presented in this study originate from inside of the workflow (per-company, where are they first inserted?). If the origin of each element can be located and then the element traced as it goes through the workflow, a better understanding of specifically who touches or needs each piece of information can be gathered. With this new knowledge, a breakdown of information by department in a company can be formed, and the consolidation of information can be implemented to prevent unnecessary encounters with information. For example, a soap sales representative probably 
never needs to see the dimensions of the soap box they develop. They simply need to know what shape it is, color, etc.

One other gap in this paper comes from the IDEF0 diagrams created to exemplify the workflows studied. Although two definitions of each workflow were created, there is a lot of work to be done on how those workflows operate and the data that is shared inside of them. By no means are the eight IDEF0 diagrams above an end-game representation. Rather, they are a means to an end in that they can be further refined. Each activity can be decomposed and extrapolated further. The opportunity created from this is therefore embedded in research and analysis of industry. Further research needs to be done on the representation these IDEF0 diagrams can take and the information that goes inside of them. Without this, the IDEF0 diagrams provide a great start, but may not be relevant in a constantly changing atmosphere.

\section{Conclusions and Future Work}

After the conclusion of Stage Three and the revisions to the IDEF0 diagrams were completed, all results from the three separate stages were compiled and reviewed. The identification and understanding of the common information model and the minimum information model is crucial to not only being able to understand which information falls into which model, but also to understanding which information elements should be captured to give a model-based definition the equivalent information content as a drawing. The common information model has been defined as the overarching, shared information between all workflows and process, across all stages of the lifecycle. The minimum information model then is the information utilized only in a specific workflow to help accomplish tasks or processes. The information utilized in the MIM is not spread throughout the lifecycle, and is often unique to the workflow it was utilized inside of. The CIM and the MIM are both views of a model-based definition and work together to define MBD. Understanding which information is necessary at specific stages of a lifecycle, or even inside of specific workflows, will improve the quality of data flow throughout a company or organization. This research has presented findings representative of the minimum information model, the common information elements, MBD and MBE. To successfully adopt MBD methods, an understanding of the information inside of a company and how that information moves is critical. Once this understanding is reached, the MIM and CIM for that company can be identified and MBE can begin to emerge as the digital architecture is created. Without knowledge of what information is necessary inside of a lifecycle and when, a true MBE will never be reached.

The first and most obvious result was that adopting MBD methods and tools is a challenge to personnel, to infrastructure, and to corporate processes and practices. To properly migrate to a digital architecture and leverage the information and competitive advantages provided by MBD methods and tools in a model-based enterprise, adequate, stable, and federated infrastructure needs to be in place. Further analysis of the IDEF diagrams (e.g., Level 1, Level $1 \mathrm{x}$, etc.) could better illustrate the depth of meaning and information impacts of the minimum information and the accompanying infrastructure, leading to enhanced understanding of the interpersonal and person-machine exchange of information in an organization. That infrastructure needs to include PLM software systems, ERP systems, translation and validation 
systems, and a network architecture that disseminates digital product data in an efficient and secure manner. Activities such as collaboration using model-based data to communicate, or production and inspection processes driven by an MBD data set, may become commonplace in a modern model-based enterprise.

To leverage the monetary investment in software and hardware infrastructure, and any costs associated with MBD creation or lifecycle data capture, training of relevant personnel needs to happen and experience with both mediums needs to grow. This training is limited by the culture and willingness to change of the company, and can be further limited by the lack of resources for employees needing to leverage MBD information. The digital architecture of a company adopting MBD must change drastically to compensate for the digitalization and dissemination of previously physical information.

An accompanying result to the monetary investment was the fact that understanding the MIM and CIM will help alleviate the stress of adopting MBD/MBE. Some companies may not need to make the transition to $\mathrm{MBD} / \mathrm{MBE}$, in fact, it may hurt them to even try due to their overall lack of preparedness and the up-front costs associated. While much of industry is trying to move towards or at least understand $\mathrm{MBD} / \mathrm{MBE}$, not all companies will need to switch or divert resources to do so. Knowing what you need to know is the first activity to understanding if $\mathrm{MBD} / \mathrm{MBE}$ is right for the company. What is clear from the IDEF0 diagrams is a relationship exists between the process activities included and the information elements associated with them. Considering evolving CAD and model-data-exchange capabilities, future work needs to focus on the relative importance of the information elements and their relationship in the context of an IDEF1 (information modeling) and IDEF1x (data modeling) structures. 


\section{References}

1. ASME, American Society of Mechanical Engineers, 2003 "Digital Product Definition Data Practices”, vol. ASME Y14.41-2003, American Society of Mechanical Engineers, 91 New York, pp. viii.

2. Camba, J. D., \& Contero, M., 2015, “Assessing the Impact of Geometric Design Intent Annotations on Parametric Model Alteration Activities,” Computers in Industry, 71, pp. 35-45.

3. Regli, W., Rossignac, J., Shapiro, V., \& Srinivasan, V. (2016). The new frontiers in computational modeling of material structures. Computer-Aided Design, 77, 73-85. doi: 10.1016/j.cad.2016.03.002

4. Hedberg Jr, T. D., Hartman, N. W., Rosche, P., \& Fischer, K. (2017). Identified research directions for using manufacturing knowledge earlier in the product life cycle. International Journal of Production Research, 55(3), 819-827. doi:10.1080/00207543.2016.1213453

5. Shannon, C. E. (1948). A mathematical theory of communication. The Bell System Technical Journal, 27, 379-423.

6. Miller, A.M., Hartman, N.W., Hedberg, T.D., Barnard-Feeney, A., Zahner, J. (2017). Towards identifying the elements of a minimum information model for use in a modelbased definition. Proceedings of the ASME 2017 12th International Manufacturing Science and Engineering Conference MSEC2017, June 4-8, 2017, Los Angeles, CA, USA.

7. Hedberg Jr, T. D., Lubell, J., Fischer, L., Maggiano, L., \& Barnard Feeney, A. (2016). Testing the Digital Thread in Support of Model-Based Manufacturing and Inspection. Journal of Computing and Information Science in Engineering, 16(2), 1-10. doi:10.1115/1.4032697

8. Louhichi, B., \& Rivest, L. 2014, "Maintaining consistency between CAD elements in collaborative design using association management and propagation.” Computers in Industry, 65(1), 124-135.

9. French, T.E., Svenson, C.L., Helsel, J.D., Urbanick, B., 1990, “Mechanical Drawing: CAD Communications”, 11th ed., McGraw-Hill, New York, NY, pp. 26.

10. Dori, D., \& Tombre, K., 1995, "From engineering drawings to 3D CAD models: are we ready now?” Computer-Aided Design, 27(4), 243-254.

11. Bechky, B. A., 2003, “Object Lessons: Workplace Artifacts as Representations of Occupational Jurisdiction1”, American Journal of Sociology, 109(3), pp. 720-752.

12. Morey, B. 2014, “Interpreting the Language of GD\&T in Metrology”, Manufacturing Engineering, 153(3), pp. 105-+.

13. Louhichi, B., \& Rivest, L. 2014, "Maintaining consistency between CAD elements in collaborative design using association management and propagation.” Computers in Industry, 65(1), 124-135.

14. Dong, A., \& Agogino, A. M. (1998). "Managing design information in enterprise-wide CAD using smart drawings”, Computer-Aided Design, 30(6), pp. 425-435. 
15. Aymar Nkondo Dika, A., Ducellier, G., Eynard, B., Lafon, P., \& Deneux, D. (2008). From workflow specification to implementation: an industrial use case. Proceedings of the 10th International Design Conference - Design 2008, (December 2014), 893-900.

16. Lin, Z. C., \& Chow, J. J. (2001). Integration Planning Model of IDEF0 and STEP Product Data Representation Methods in a CMM Measuring System. International Journal of Advanced Manufacturing Technology, 17, 39-53.

17. National Institute of Standards and Technology. FIPS PUB 183: Integration Definition for Function Modeling (IDEF0). Gaithersburg, MD, USA: National Institute of Standards and Technology.

18. ISA, 2013, "Enterprise-Control System Integration Part 3: Activity Models of Manufacturing Operations Management," ISA, North Carolina.

19. ISA, 2012, "Enterprise-Control System Integration Part 4: Objects and attributes for manufacturing operations management integration," ISA, North Carolina.

20. Feng, S. C., 1994, "Dimensional Inspection Planning Based on Product Data Standards," National Institute of Standards and Technology.

21. Pugh, S., 1991, Total design: integrated methods for successful product engineering, Addison-Wesley Pub. Co., Wokingham, England; Reading, Mass.

22. Dalkey, Norman; Helmer, Olaf (1963). "An Experimental Application of the Delphi Method to the use of experts". Management Science. 9 (3): 458-467. doi:10.1287/mnsc.9.3.458.

23. International Standards Organization. (2008). Document management -- Portable document format -- Part 1: PDF 1.7 (Vol. ISO 32000-1): ISO/TC 171/SC 2.

24. International Standards Organization. (2014). Document management -- 3D use of Product Representation Compact (PRC) format -- Part 1: \{PRC\} 10001 (Vol. ISO 147391): ISO/TC 171/SC 2.

25. International Standards Organization. (2014). Industrial automation systems and integration -- Product data representation and exchange -- Part 242: Application protocol: Managed model-based 3D engineering (Vol. ISO 10303-242): ISO/TC 184/SC 4.

26. International Standards Organization. (2012). Industrial automation systems and integration -- JT file format specification for 3D visualization (Vol. ISO 14306): ISO/TC 184/SC 4.

27. Ruemler, S.P., Zimmerman, K., Hartman, N.W., Hedberg, T.D., Barnard-Feeney, A. (2016). "Promoting model-based definition to establish a common information model." Journal of Manufacturing Science and Engineering, 139 (5). doi:10.1115/1.4034625

28. Adamski, W., 2010, "Adjustment and Implementation of CAD/CAM Systems Being Used in Polish Aviation Industry,” Journal of Machine Engineering, 10(3), pp. 37-47.

29. Alemanni, M., F. Destefanis, and E. Vezzetti. "Model-based definition design in the product lifecycle management scenario." The International Journal of Advanced Manufacturing Technology 52.1-4 (2011): 1-14. 


\section{Appendix A: Survey Questions for Stage One}

1. Which industry sector(s) does your company identify with (check all that apply)?
a. Aerospace
b. Defense/Military
c. Automotive
d. Consumer products
e. Medical
f. Energy/Utilities
g. Other

2. How many employees are there in your company?
a. $0-50$
b. $51-250$
c. $251-500$
d. 501 or more

3. Where are you (the person filling out the survey) located?
a. U.S.
b. Outside U.S.

4. Which region of the U.S. are you from? If $\mathbf{3}$ a
a. Northwest region
b. Southwest region
c. Southeast region
d. Northeast region
e. Midwest region

5. Where are you (the person filling out the survey) located outside the U.S.? If $\mathbf{3} \mathbf{b}$
a. North America (Not U.S.)
b. South America
c. Asia
d. Europe
e. Africa
f. Oceania (Australia or South Pacific)

6. Which of the following best identifies your current job area within the company (select only one)?
a. Sales
b. Design engineer/designer
c. Analysis 

d. Manufacturing/production
e. Supply chain/supplier management
f. Quality/inspection
g. Product service/support
h. Management
i. Other

7. Based on your answer to the previous question, which of the following best describes the format or medium in which product information comes to you for use?
a. 2D paper drawings
b. 2D electronic drawings
c. 3D native CAD models
d. Derivative of 3D CAD model (STEP, JT, 3D PDF, etc.)
e. Other

8. Which workflow do you most actively participate in? (select one)
a. Concept to Prototype
b. Prototype to Detailed Product Definition
c. Detailed Product Definition to Manufacturing/Production
d. Manufacturing/Production to Inspection
e. I do not actively participate in these workflows

9. What type of information is created or used in the Concept to Prototype workflow? (select all that apply)
a. Product specifications
b. Performance criteria
c. Dimensional information
d. Materials specification/selection
e. Surface treatment
f. Manufacturing methods
g. Basic object geometry
h. Other

10. Are drawings currently used to communicate this information in your process?
a. Yes, drawings are used exclusively.
b. Yes, but with other accompanying documents.
c. No

11. What other things are used to communicate this information? (Select all that apply)
a. Corporate Standards
b. Test Documents 
c. Specification Documents

d. Process Plans

e. Contracts

f. Other

12. In your company, could the items in Question 9 be communicated via a 3D model of the product instead of a drawing?
a. Yes
b. No

13. What prevents models from being used to communicate information in this workflow? (Select all that apply)
a. Models are too cumbersome to modify and interrogate at this stage of the lifecycle.
b. The information does not easily take a form that is useful in a model at this stage of the lifecycle.
c. It takes too much time to enter this information into a model
d. Uncertainty about changes in software and accessibility of data in the future prevent us from using models at this stage of the lifecycle.
e. The functionality of the CAD tool does not readily accommodate this type of information at this stage of the lifecycle.
f. Other

14. What type of information is created or used in the Prototype to Detailed Product Definition workflow? (select all that apply)
a. Performance characteristics
b. refined object geometry
c. Dimensional information
d. Tolerance information
e. Materials specification/selection
f. Materials models
g. Manufacturing process models
h. Surface treatment
i. Manufacturing methods
j. Revision/version history
k. Test results
I. Other

15. Are drawings currently used to communicate this information in your process?
a. Yes, drawings are used exclusively.
b. Yes, but with other accompanying documents. 
c. No

16. What other things are used to communicate this information? (Select all that apply)

a. Corporate standards

b. Test documents

c. Specification documents

d. process plans

e. Contracts

f. Internal memo

g. Test Lab reports

h. Design reviews

i. Other

17. In your company, could the items in Question 9 be communicated via a 3D model of the product instead of a drawing?
a. Yes
b. No

18. What prevents models from being used to communicate information in this workflow? (Select all that apply)

a. Models are too cumbersome to modify and interrogate at this stage of the lifecycle.

b. The information does not easily take a form that is useful in a model at this stage of the lifecycle.

c. It takes too much time enter this information into a model.

d. Uncertainty about changes in software and accessibility of data in the future prevent us from using model at this stage of the lifecycle.

e. The functionality of the CAD tool does not readily accommodate this type of information at this stage of the lifecycle.

f. Other

19. What type of information is created or used in the Detailed Product Definition to Manufacturing workflow? (select all that apply)
a. Detailed product geometry
b. Final dimensional information
c. Tolerance information/GDT
d. Materials definition
e. Manufacturing process models
f. Finished surface characteristics
g. Manufacturing methods
h. Revision/version history 
i. Supplier data

j. Inspection data

k. Other

20. Are drawings currently used to communicate this information in your process?
a. Yes, drawings are used exclusively.
b. Yes, but with other accompanying documents.
c. No

21. What other things are used to communicate this information? (Select all that apply)
a. Corporate standards
b. Industry standards
c. Test documents
d. Specification documents
e. Process plans
f. Contracts
g. Internal memo
h. Test Lab reports
i. Design reviews
j. Other

22. In your company, could the items in the previous question be communicated via a $3 \mathrm{D}$ model of the product instead of a drawing?
a. Yes
b. No

23. What prevents models from being used to communicate information in this workflow? (Select all that apply)
a. Models are too cumbersome to modify and interrogate at this stage of the lifecycle.
b. The information does not easily take a form that is useful in a model at this stage of the lifecycle.
c. It takes too much time to enter this information into a model.
d. Uncertainty about changes in software and accessibility of data in the future prevent us from using models at this stage of the lifecycle.
e. The functionality of the CAD tool does not readily accommodate this type of information at this stage of the lifecycle.
f. Other (free response field)

24. What type of information is created or used in the Manufacturing to Inspection workflow? (select all that apply) 

a. Detailed product geometry
b. Final dimensional information
c. Tolerance information/GDT
d. Materials definition
e. Finished surface characteristics
f. Machine tool process capability
g. Manufacturing methods
h. Validation certificates
i. Revision/version history
j. Supplier process capability data
k. Other

25. Are drawings currently used to communicate this information in your process?
a. Yes, drawings are used exclusively.
b. Yes, but with other accompanying documents.
c. No

26. What other things are used to communicate this information? (Select all that apply)
a. Corporate standards
b. Industry standards
c. Specification documents
d. Process plans
e. Contracts
f. Internal memo
g. Test Lab reports
h. Other

27. In your company, could the items in Question 8 be communicated via a 3D model of the product instead of a drawing?
a. Yes
b. No

28. What prevents models from being used to communicate information in this workflow? (Select all that apply)
a. Models are too cumbersome to modify and interrogate at this stage of the lifecycle.
b. The information does not easily take a form that is useful in a model at this stage of the lifecycle.
c. Uncertainty about changes in software and accessibility of data in the future prevent us from using models at this stage of the lifecycle.


d. The functionality of the CAD tool does not readily accommodate this type of information at this stage of the lifecycle.

e. Other

29. Is there any additional information you would like to provide regarding the use of CAD models in lieu of drawings in the aforementioned workflows? (free response field)

30. Optional: Enter your name and email address if you would like a copy of the final results (free response fields) 


\section{Appendix B: Delphi Study Round 1 Questions}

1. Which industry sector best represents your company or the division of the company where you work?
a. Aerospace
b. Defense/Military
c. Automotive
d. Consumer products
e. Heavy equipment
f. Industrial machines
g. Medical device/equipment
h. Energy/Utilities
i. Other

2. How many employees are there in your company?
a. 1-4
b. 5-9
C. $10-19$
d. 20-49
e. 50-99
f. $100-249$
g. 250-499
h. $500+$

3. Where are you located (the person completing the survey)?
a. U.S.
b. Outside U.S.

4. Please select your region (the person completing the survey) (Continued)
a. Northwestern Region
b. Southwestern Region
c. Southeastern Region
d. Northeastern Region
e. Midwestern Region

5. Please select your country or region outside the U.S. (the person completing the survey).
a. Brazil
b. China
c. India
d. Mexico 

e. Russia
f. Asia (not China)
g. Europe
h. South America

6. What is your primary role within the company?
a. Sales
b. Order processing
c. Procurement/Supply chain
d. Engineer/analysis
e. Engineer/designer
f. Engineer/Project Engineer
g. Engineer/Systems Engineer
h. Production/planning
i. Production/operation
j. Quality/inspection
k. Product service/support
l. Management
m. Other

7. What workflow do you most commonly participate in?
a. Detailed Design to Design/Make
b. Detailed Design to Make-to-Print (Make-to-Model)
c. Detailed Design to Process Planning
d. Detailed Design to Work Instruction
e. Requirements to Design/Make
f. Requirements to Make-to-Print (Make-to-Model)
g. Design/Make to Maintenance
h. Design/Make to Repair
i. Design/Make to Overhaul
j. Make-to-Print (Make-to-Model) to Maintenance
k. Make-to-Print (Make-to-Model) to Repair
l. Make-to-Print (Make-to-Model) to Overhaul
m. Concept Design to Design Review
n. Design Review to Requirements Review
o. Machining to Quality Control/Inspection
p. Casting to Quality Control/Inspection
q. In Use to Maintenance
r. In Use to Repair
s. In Use to Overhaul

8. Based on your answer to Q7, which of the following elements are necessary to successfully communicate within the workflow? Select all that apply.

a. 3D Geometry 
b. Dimensional Information

c. Detailed Product Geometry

d. Revision/Version history

e. GD\&T

f. Materials Specifications/Definition

g. Surface Finish/Characteristics

h. Manufacturing Methods

i. Machine Tool Process Capability

j. Performance Characteristics

k. Test Results

l. Supplier Data

m. Company/Industry Specific Interpretation Specifications

n. Manufacturing and Inspection Rules

o. Engineering Notes

p. Bill of Materials

q. Provenance Information

r. Industry/Regulatory/Company Traceability Data

s. Referenced/Related Appropriate Standards

t. Inspection Reports/Data

u. Manufacturing Data, Metrics, KPIs for Process Control and Improvement

v. Change Management Data

w. Other

9. If you had to identify your role as primarily an author of model-based definition information, or a consumer of model-based definition information, which one would it be?
a. Author
b. Consumer

10. Would you consider your company (or the division in which you work) an OEM or a supplier?
a. Supplier
b. OEM

If "a”, go to Q11

If "b", go to Q12

11. If your company (or the division in which you work) is a supplier to another company, would you consider your company as primarily a design-make supplier or a make-to-print (or maketo-model) company?

a. Design-make

b. Make-to-print (make-to-model) 
12. To what extent does your job role involve the use of a drawing or model to complete your job effectively?
a. 10 - I use a drawing or a model-based definition daily.
b. 9
c. 8
d. 7
e. 6
f. 5 - I sometimes use a drawing or a model-based definition.
g. 4
h. 3
i. 2
j. 1 - I never use a drawing or a model-based definition.

13. Which of the following best represents the form of product definition data you utilize to perform your job? (select one item)
a. Drawings only
b. Primarily drawings (with supplemental models)
c. Primarily models (with supplemental drawings)
d. 3D models only

14. Do you utilize neutral file formats?
a. Yes
b. No

15. Which CAD-derivative file formats do you use? (Select all that apply)
a. IGES
b. JT
c. Parasolid (.XT)
d. PRC
e. STEP
f. Stereolithography (.STL)
g. 2D PDF
h. 3D PDF
i. VRML
j. Other

16. In order to promote the use of a model-based definition, if a native CAD model needs to be translated to another format, which pieces of information in that file are critical to surviving translation?
a. 3D Geometry
b. Dimensional Information
c. Detailed Product Geometry
d. Revision/Version History
e. GDT
f. Materials 
g. Manufacturing Methods

h. Company/Industry Specific Interpretation Specifications

i. Engineering Notes

j. Bill of Materials

k. Provenance Information

l. Traceability Data

m. Inspection Reports

n. References/Related/Appropriate Standards

o. Inspection Reports/Data

p. Other

17. Based on your experience, what information is commonly lost when converting a model to a neutral file format?

a. 3D Geometry

b. Dimensional Information

c. Detailed Product Geometry

d. Revision/Version history

e. GDT

f. Materials Specifications/Definition

g. Surface Finish/Characteristics

h. Manufacturing Methods

i. Machine Tool Process Capability

j. Performance Characteristics

k. Test Results

l. Supplier Data

m. Company/Industry Specific Interpretation Specifications

n. Manufacturing and Inspection Rules

o. Engineering Notes

p. Bill of Materials

q. Provenance Information

r. Industry/Regulatory/Company Traceability Data

s. Referenced/Related Appropriate Standards

t. Inspection Reports/Data

u. Manufacturing Data, Metrics, KPIs for Process Control and Improvement

v. Change Management Data

w. Other

18. Do people in your company create $3 \mathrm{D}$ models from existing $2 \mathrm{D}$ drawings?
a. Yes
b. No

19. Assuming you use a model-based definition across the lifecycle of your products, which elements are common from one lifecycle stage to the next? Select all that apply.

a. 3D Geometry

b. Dimensional Information 
c. Detailed Product Geometry

d. Revision/Version history

e. GDT

f. Materials Specifications/Definition

g. Surface Finish/Characteristics

h. Manufacturing Methods

i. Machine Tool Process Capability

j. Performance Characteristics

k. Test Results

l. Supplier Data

m. Company/Industry Specific Interpretation Specifications

n. Manufacturing and Inspection Rules

o. Engineering Notes

p. Bill of Materials

q. Provenance Information

r. Industry/Regulatory/Company Traceability Data

s. Referenced/Related Appropriate Standards

t. Inspection Reports/Data

u. Manufacturing Data, Metrics, KPIs for Process Control and Improvement

v. Change Management Data

w. Other

20. Please specify any questions you have or any information you think we missed that would be relevant for us to gather, or any suggestions you may have for our future surveys:

We would like to thank you once again for being a part of our Delphi Study. As we stated, this is a multi-round survey and would like you to participate in all stages of data collection.

Please navigate to the link below in order to provide contact information for distribution of round two. Thank you!

https://purdue.qualtrics.com/SE/?SID=SV_5vwqM734OKGdscB 


\section{Appendix C: Delphi Round Two}

1. Which industry sector best represents your company or the division of the company where you work?
j. Aerospace
k. Defense/Military
l. Automotive
m. Consumer products
n. Heavy equipment
o. Industrial machines
p. Medical device/equipment
q. Energy/Utilities
r. Other

2. What is your primary role within the company?
n. Sales
o. Order processing
p. Procurement/Supply chain
q. Engineer/analysis
r. Engineer/designer
s. Engineer/Project Engineer
t. Engineer/Systems Engineer
u. Production/planning
v. Production/operation
w. Quality/inspection
x. Product service/support
y. Management
z. Other

3. What workflow do you most commonly participate in?
a. Detailed Design to Design/Make
b. Detailed Design to Make-to-Print (Make-to-Model)
c. Detailed Design to Process Planning
d. Detailed Design to Work Instruction
e. Requirements to Design/Make
f. Requirements to Make-to-Print (Make-to-Model)
g. Design/Make to Maintenance
h. Design/Make to Repair
i. Design/Make to Overhaul
j. Make-to-Print (Make-to-Model) to Maintenance
k. Make-to-Print (Make-to-Model) to Repair 
l. Make-to-Print (Make-to-Model) to Overhaul

m. Concept Design to Design Review

n. Design Review to Requirements Review

o. Machining to Quality Control/Inspection

p. Casting to Quality Control/Inspection

q. In Use to Maintenance

r. In Use to Repair

s. In Use to Overhaul

t. Other

The following questions will be used to identify the most critical pieces of information that should be included in a model-based definition for a given workflow. At a minimum, which of the following should be included in the MBD for the workflow you participate in? Please rank each item (1 - not critical, 7 - most critical).

\section{3D Geometry}

$\begin{array}{lllllll}1 & 2 & 3 & 4 & 5 & 6 & 7\end{array}$

5. Dimensional Information

$\begin{array}{lllllll}1 & 2 & 3 & 4 & 5 & 6 & 7\end{array}$

6. Detailed Product Geometry

$\begin{array}{llllllll}1 & 2 & 3 & 4 & 5 & 6 & 7\end{array}$

7. Revision/Version History

$\begin{array}{lllllll}1 & 2 & 3 & 4 & 5 & 6 & 7\end{array}$

8. Geometric Dimensions and Tolerances (GD\&T)

$\begin{array}{lllllll}1 & 2 & 3 & 4 & 5 & 6 & 7\end{array}$

9. Materials Specifications/Definitions

$\begin{array}{llllllll}1 & 2 & 3 & 4 & 5 & 6 & 7\end{array}$

10. Surface Finish/Characteristics

$\begin{array}{lllllll}1 & 2 & 3 & 4 & 5 & 6 & 7\end{array}$

\section{Manufacturing Methods}

$\begin{array}{llllllll}1 & 2 & 3 & 4 & 5 & 6 & 7\end{array}$

12. Machine Tool Process Capability

$\begin{array}{lllllll}1 & 2 & 3 & 4 & 5 & 6 & 7\end{array}$

\section{Performance Characteristics

$\begin{array}{lllllll}1 & 2 & 3 & 4 & 5 & 6 & 7\end{array}$

14. Test Results 


$\begin{array}{lllllll}1 & 2 & 3 & 4 & 5 & 6 & 7\end{array}$

\section{Supplier Data}

$\begin{array}{lllllll}1 & 2 & 3 & 4 & 5 & 6 & 7\end{array}$

16. Company/Industry Specific Interpretation Specifications

$\begin{array}{lllllll}1 & 2 & 3 & 4 & 5 & 6 & 7\end{array}$

17. Manufacturing and Inspection Rules

$\begin{array}{llllllll}1 & 2 & 3 & 4 & 5 & 6 & 7\end{array}$

18. Engineering Notes

$\begin{array}{llllllll}1 & 2 & 3 & 4 & 5 & 6 & 7\end{array}$

19. Bill of Materials

$\begin{array}{lllllll}1 & 2 & 3 & 4 & 5 & 6 & 7\end{array}$

20. Provenance Information

$\begin{array}{llllllll}1 & 2 & 3 & 4 & 5 & 6 & 7\end{array}$

21. Industry/Regulatory/Company Traceability Data

$\begin{array}{lllllll}1 & 2 & 3 & 4 & 5 & 6 & 7\end{array}$

22. Referenced/Related Appropriate Standards

$\begin{array}{lllllll}1 & 2 & 3 & 4 & 5 & 6 & 7\end{array}$

23. Inspection Reports/Data

$\begin{array}{llllllll}1 & 2 & 3 & 4 & 5 & 6 & 7\end{array}$

24. Manufacturing Data/Metrics/KPIs for Process Control and Improvement

$\begin{array}{lllllll}1 & 2 & 3 & 4 & 5 & 6 & 7\end{array}$

25. Change Management Data

$\begin{array}{lllllll}1 & 2 & 3 & 4 & 5 & 6 & 7\end{array}$

\section{Product Specifications}

$\begin{array}{lllllll}1 & 2 & 3 & 4 & 5 & 6 & 7\end{array}$

The next set of questions will be used to identify the common information elements across the lifecycle. Please rank the following items with regard to their importance to a model-based definition across the lifecycle regardless of workflow. (1 - not important, 7 - most important).

\section{3D Geometry}




$\begin{array}{lllllll}1 & 2 & 3 & 4 & 5 & 6 & 7\end{array}$

28. Dimensional Information

$\begin{array}{llllllll}1 & 2 & 3 & 4 & 5 & 6 & 7\end{array}$

29. Detailed Product Geometry

$\begin{array}{llllllll}1 & 2 & 3 & 4 & 5 & 6 & 7\end{array}$

30. Revision/Version History

$\begin{array}{llllllll}1 & 2 & 3 & 4 & 5 & 6 & 7\end{array}$

31. Geometric Dimensions and Tolerances (GD\&T)

$\begin{array}{lllllll}1 & 2 & 3 & 4 & 5 & 6 & 7\end{array}$

32. Materials Specifications/Definitions

$\begin{array}{llllllll}1 & 2 & 3 & 4 & 5 & 6 & 7\end{array}$

33. Surface Finish/Characteristics

$\begin{array}{llllllll}1 & 2 & 3 & 4 & 5 & 6 & 7\end{array}$

34. Manufacturing Methods

$\begin{array}{llllllll}1 & 2 & 3 & 4 & 5 & 6 & 7\end{array}$

35. Machine Tool Process Capability

$\begin{array}{llllllll}1 & 2 & 3 & 4 & 5 & 6 & 7\end{array}$

36. Performance Characteristics

$\begin{array}{llllllll}1 & 2 & 3 & 4 & 5 & 6 & 7\end{array}$

37. Test Results

$\begin{array}{llllllll}1 & 2 & 3 & 4 & 5 & 6 & 7\end{array}$

38. Supplier Data

$\begin{array}{llllllll}1 & 2 & 3 & 4 & 5 & 6 & 7\end{array}$

39. Company/Industry Specific Interpretation Specifications

$\begin{array}{lllllll}1 & 2 & 3 & 4 & 5 & 6 & 7\end{array}$

40. Manufacturing and Inspection Rules

$\begin{array}{llllllll}1 & 2 & 3 & 4 & 5 & 6 & 7\end{array}$

41. Engineering Notes

$\begin{array}{llrlllll}1 & 2 & 3 & 4 & 5 & 6 & 7\end{array}$

42. Bill of Materials

$\begin{array}{llllllll}1 & 2 & 3 & 4 & 5 & 6 & 7\end{array}$


43. Provenance Information

$\begin{array}{lllllll}1 & 2 & 3 & 4 & 5 & 6 & 7\end{array}$

\section{Industry/Regulatory/Company Traceability Data}

$\begin{array}{lllllll}1 & 2 & 3 & 4 & 5 & 6 & 7\end{array}$

45. Referenced/Related Appropriate Standards

$\begin{array}{lllllll}1 & 2 & 3 & 4 & 5 & 6 & 7\end{array}$

46. Inspection Reports/Data

$\begin{array}{lllllll}1 & 2 & 3 & 4 & 5 & 6 & 7\end{array}$

\section{Manufacturing Data/Metrics/KPIs for Process Control and Improvement $\begin{array}{lllllll}1 & 2 & 3 & 4 & 5 & 6 & 7\end{array}$}

48. Change Management Data

$\begin{array}{lllllll}1 & 2 & 3 & 4 & 5 & 6 & 7\end{array}$

\section{Product Specifications}

$\begin{array}{lllllll}1 & 2 & 3 & 4 & 5 & 6 & 7\end{array}$

50. Please specify any questions you have or any information you think we missed that would be relevant for us to gather, or any suggestions you may have for our future surveys.

51. We would like to thank you once again for being a part of our Delphi Study. As we stated previously, this is a multi-round survey and would like you to participate in all stages of collection data. 


\section{Appendix D: Delphi Round Three}

1. Which industry sector best represents your company or the division of the company where you work?
s. Aerospace
t. Defense/Military
u. Automotive
v. Consumer products
w. Heavy equipment
$\mathrm{x}$. Industrial machines
y. Medical device/equipment
z. Energy/Utilities
aa. Other

2. What is your primary role within the company?
aa. Sales
bb. Order processing
cc. Procurement/Supply chain
dd. Engineer/analysis
ee. Engineer/designer
ff. Engineer/Project Engineer
gg. Engineer/Systems Engineer
hh. Production/planning
ii. Production/operation
jj. Quality/inspection
kk. Product service/support
1l. Management
mm. Other

3. What workflow do you most commonly participate in?
a. Detailed Design to Design/Make
b. Detailed Design to Make-to-Print (Make-to-Model)
c. Detailed Design to Process Planning
d. Detailed Design to Work Instruction
e. Requirements to Design/Make
f. Requirements to Make-to-Print (Make-to-Model)
g. Design/Make to Maintenance
h. Design/Make to Repair
i. Design/Make to Overhaul
j. Make-to-Print (Make-to-Model) to Maintenance
k. Make-to-Print (Make-to-Model) to Repair 
l. Make-to-Print (Make-to-Model) to Overhaul

m. Concept Design to Design Review

n. Design Review to Requirements Review

o. Machining to Quality Control/Inspection

p. Casting to Quality Control/Inspection

q. In Use to Maintenance

r. In Use to Repair

s. In Use to Overhaul

t. Other

The following questions will be used to identify the most critical pieces of information that should be included in a model-based definition. Which of the following items should be included in the model-based definition? Please rate each item ( 0 - not applicable; 1 - least important through 10 - most important). Any questions not clicked on will be default recorded as a zero.

4. 3D Geometry

$\begin{array}{lllllllllll}0 & 1 & 2 & 3 & 4 & 5 & 6 & 7 & 8 & 9 & 10\end{array}$

5. Dimensional Information

$\begin{array}{lllllllllll}0 & 1 & 2 & 3 & 4 & 5 & 6 & 7 & 8 & 9 & 10\end{array}$

6. Detailed Product Geometry

$\begin{array}{lllllllllll}0 & 1 & 2 & 3 & 4 & 5 & 6 & 7 & 8 & 9 & 10\end{array}$

7. Revision/Version History

$\begin{array}{lllllllllll}0 & 1 & 2 & 3 & 4 & 5 & 6 & 7 & 8 & 9 & 10\end{array}$

8. Geometric Dimensions and Tolerances (GD\&T)

$\begin{array}{llllllllllll}0 & 1 & 2 & 3 & 4 & 5 & 6 & 7 & 8 & 9 & 10\end{array}$

9. Materials Specifications/Definitions

$\begin{array}{lllllllllll}0 & 1 & 2 & 3 & 4 & 5 & 6 & 7 & 8 & 9 & 10\end{array}$

10. Surface Finish/Characteristics

$\begin{array}{lllllllllll}0 & 1 & 2 & 3 & 4 & 5 & 6 & 7 & 8 & 9 & 10\end{array}$

11. Engineering Notes

$\begin{array}{lllllllllll}0 & 1 & 2 & 3 & 4 & 5 & 6 & 7 & 8 & 9 & 10\end{array}$

\section{Bill of Materials}

$\begin{array}{lllllllllll}0 & 1 & 2 & 3 & 4 & 5 & 6 & 7 & 8 & 9 & 10\end{array}$

13. Referenced/Related Appropriate Standards

$\begin{array}{lllllllllll}0 & 1 & 2 & 3 & 4 & 5 & 6 & 7 & 8 & 9 & 10\end{array}$




\section{Change Management Data}

$\begin{array}{lllllllllll}0 & 1 & 2 & 3 & 4 & 5 & 6 & 7 & 8 & 9 & 10\end{array}$

\section{Product Specifications}

$\begin{array}{lllllllllll}0 & 1 & 2 & 3 & 4 & 5 & 6 & 7 & 8 & 9 & 10\end{array}$

Each of these elements are required in different forms depending on industry sector, job role, workflow and requirements specified by the OEM or project lead. These elements are typically presented either in the model or in the drawing. For this section, please specify the form in which the element needs to be present:

\begin{tabular}{|l|l|l|}
\hline Information Item & In model & In drawing \\
\hline 16. SD Geometry & & \\
\hline 17. Dimensional information & & \\
\hline 18. Detailed Product Geometry & & \\
\hline 19. Revision/Version History & & \\
\hline 20. Geometric Dimensions and Tolerances (GD\&T) & & \\
\hline 21. Materials Specifications/Definitions & & \\
\hline 22. Surface Finish/Characteristics & & \\
\hline 23. Engineering Notes & & \\
\hline 24. Bill of Materials & & \\
\hline 25. Referenced/Related Appropriate Standards & & \\
\hline 26. Change Management Data & & \\
\hline 27. Product Specifications & & \\
\hline
\end{tabular}

28. if you could only select one item, which do you believe is the most important data item? (select only one)
a) 3D Geometry
b) Dimensional Information
c) Detailed Product Geometry
d) Revision/Version History
e) Geometric Dimensions and Tolerances (GD\&T)
f) Materials Specifications/Definitions
g) Engineering Notes
h) Bill of Materials
i) Referenced/Related Appropriate Standards
j) Change Management Data
k) Surface Finish/Characteristics
l) Product Specifications

Please answer each of the following questions using the given scale. ( 0 - not applicable; 1 - least important through 10 - most important). Any questions not clicked on will be default recorded as a zero. 
29. To what extent is education a factor in the ability of a company to adopt MBD/MBE?
0
12
3
$4 \quad 5 \quad 6$
$7 \quad 8$
$8 \quad 9 \quad 10$

30. To what extent is corporate culture a factor in the ability of a company to adopt MBD/MBE?
0

\begin{abstract}
1
\end{abstract}
2
3
4
5
6
7
89
10

31. To what extent does the availability of proper technology infrastructure affect the adoption of MBD/MBE?

$\begin{array}{lllllllllll}0 & 1 & 2 & 3 & 4 & 5 & 6 & 7 & 8 & 9 & 10\end{array}$

32. To what extent does having a relevant data architecture affect the adoption of MBD/MBE?

$\begin{array}{lllllllllll}0 & 1 & 2 & 3 & 4 & 5 & 6 & 7 & 8 & 9 & 10\end{array}$

33. Please specify any questions you have or any information you think we missed that would be relevant for us to gather.

We would like to thank you once again for being a part of our research project. As we stated previously, this is the final round of data collection. Thank you! 


\title{
Appendix E: Interview Questions
}

1. What is your industry sector (Ex. Aerospace, Medical, Defense/Military...)?

2. What is your job role (Ex. Sales, Product Service/Support, Management...)?

3. Which workflow do you primarily participate in?

\author{
Example Workflows: \\ Detailed Design to Design/Make \\ Detailed Design to Make-to-Print (Make to Model) \\ Detailed Design to Process Planning \\ Detailed Design to Work Instruction \\ Requirements to Design/Make \\ Requirements to Make-to-Print (Make-to-Model) \\ Design/Make to Maintenance \\ Design/Make to Repair \\ Design/Make to Overhaul \\ Make-to-Print to Maintenance \\ Make-to-Print to Repair \\ Make-to-Print to Overhaul \\ Concept Design to Design Review \\ Design Review to Requirements Review \\ Machining to Quality Control/Inspection \\ Casting to Quality Control/Inspection \\ In Use to Maintenance \\ In Use to Repair \\ In Use to Overhaul \\ Other
}

Our survey asked participants to rate the importance of specific elements inside of and outside of workflows. Elements that were top-rated and above the cutoff threshold are being kept for our round 3 data. Here is a list of the most-important responses from the survey:

3D Geometry

Dimensional Information

Detailed Product Geometry

Revision/Version History

Geometric Dimensions and Tolerances (GD\&T)

Materials Specifications/Definitions

Engineering Notes

Bill of Materials

Referenced/Related Appropriate Standards

Change Management Data

Surface Finish/Characteristics 


\section{Product Specifications}

4. To what extent is your own job experience reflective of this list?

a. Are there any items missing from or that shouldn't be on this list?

Your industry sector provides you with a niche environment where specific information is needed based on your job role. For example, someone working in sales in the aerospace sector may need access to tolerance information, head indexes, etc. Whereas someone working in sales in the medical field may need access to surface finish and detailed product geometry.

5. From the list, what elements would you say are imperative to your job role?

6. What information is irrelevant to you or your job role?

7. According to the workflow you follow, what information must be present?

8. How does the job role you identified fit into the workflow that you're involved in? (Ex. Are you at the start of the workflow or somewhere in the middle/end? What information is ONLY passed on from your role? What information is ONLY passed to your role? What information is only relevant to your role?)

9. As best you can, take the items from the list above and specify why they matter or don't matter to your industry sector.

a. Now, bring it down to the job role level and specify why they matter or don't matter to your job role.

b. Finally, of the items that you've selected, specify how they fit into the workflow you're a part of.

10. Previous surveys have shown that one of the biggest reasons for not using models instead of drawings is that information in an MBD does not easily take a form that is useful in a given workflow. Why do you think this is?

11. Information from previous surveys have also shown that when asked to choose what form of product definition is utilized to perform their job, the majority of answers are in the "Primary Models (with supplemental drawings) category. Why do you think this is the most commonly utilized form of product definition?

For clarity, the full list of options that participants could choose from and their response percentages are listed below:

Drawings only: 0\% 
Primarily Drawings (with supplemental models): 29\%

Primarily Models (with supplemental drawings): 42\%

3D Models Only: 29\% 